\title{
A QUALITATIVE EXPLORATION OF HEALTH BELIEFS AMONG MIDLIFE FILIPINO AMERICAN WOMEN IN CALIFORNIA
}

\author{
A Master's Thesis \\ Presented to the Faculty of California Polytechnic State University \\ San Luis Obispo
}

In partial fulfillment

of the requirements for the degree

Master of Science in Kinesiology

By

Lorneneth Merana

May 1, 2008 


\section{AUTHORIZATION FOR REPRODUCTION OF MASTER'S THESIS}

I grant permission for the reproduction of this thesis in its entirety or any of its parts without further authorization from me.
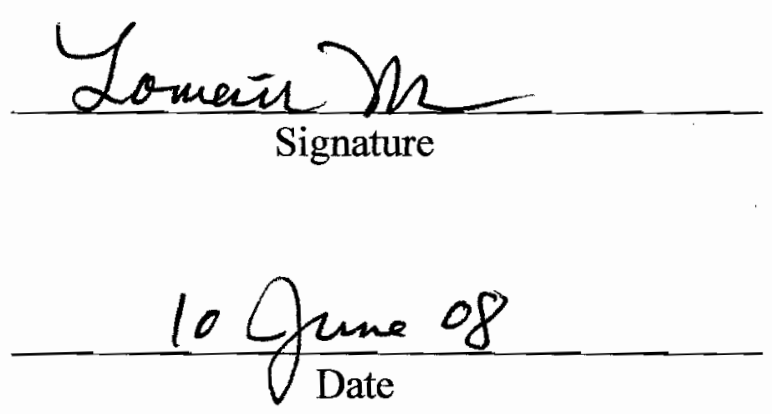


\section{APPROVAL PAGE}

TITLE:

A Qualitative Exploration of Health Beliefs among

Midlife Filipino American Women in California

AUTHOR:

Lorneneth Merana

DATE SUBMITTED:

May 1, 2008

Dr. Camille O'Bryant

Advisor/Committee Chair

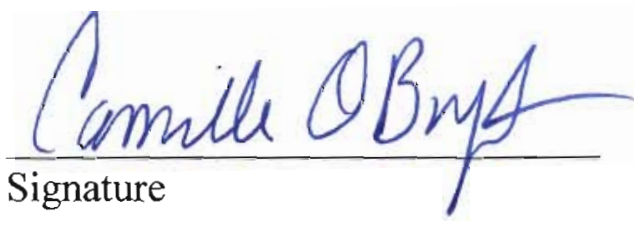

Dr. Kris Jankovitz

Committee Member

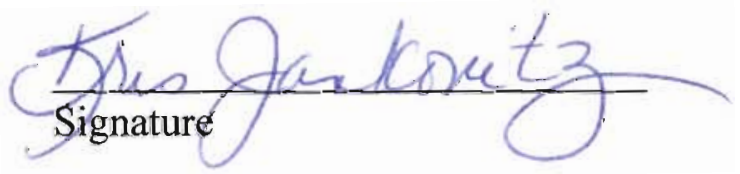

Dr. Pat Hosegood-Martin

Committee Member

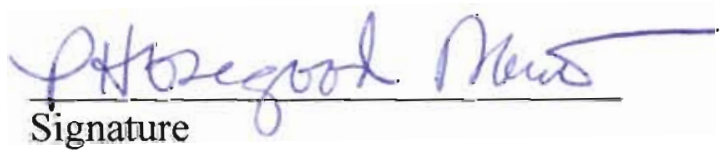




\section{ABSTRACT \\ A QUALITATIVE EXPLORATION OF HEALTH BELIEFS AMONG MIDLIFE FILIPINO AMERICAN WOMEN IN CALIFORNIA}

Lorneneth Merana

Research pertaining to Filipino American health issues is growing but most remain delimited to children, college students, and older adults. The purpose of this study was two-fold. The first was to define the health beliefs of four Filipino American women who were in midlife through in-depth one-on-one interviews regarding cardiovascular diseases and in utilizing health care to prevent, diagnose, or treat those diseases. The second purpose was to conceptualize potential links between the health beliefs of those Filipino American women and their attitudes or choices about health care utilization.

Conclusively, the health beliefs of the four midlife Filipino-American women in this study were firmly established in their self-efficacy in recognizing their risks of cardiovascular disease and in making the best choices to decrease their risk of developing one. In contrast to the assumptions of this study, I found that these women a) were very aware of the resources available to them when they had health issues and b) their health beliefs regarding cardiovascular diseases did not hinder utilization of the health care services to which they had access. In fact, they were more receptive to utilizing health care resources to garner a medical perspective as long as they gained a clearer understanding of the health issue and that the treatment options were as natural as possible. 


\section{ACKNOWLEDGEMENTS}

Many thanks to my parents, Alex and Lorna, and to my thesis advisors for never letting me sink in the roughest of seas. A special acknowledgement to my participants who showed patience and a willingness to share their lives with a member of the next generation of Filipino American women. 
Table of Contents

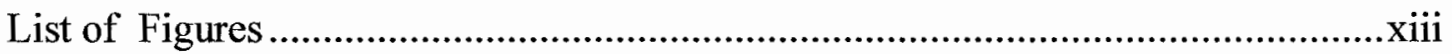

\section{Chapter 1}

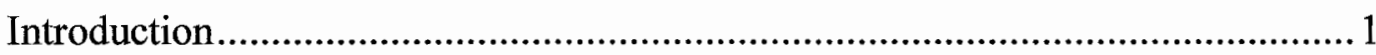

Background of the Study ......................................................... 1

Statement of the Problem and Purpose .................................................... 3

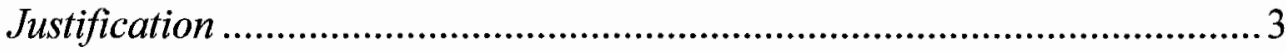

Assumptions ............................................................................. 3

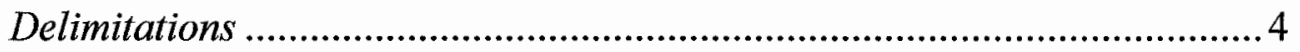

Limitations.............................................................................4

Research Question .................................................................... 5

Definition of Terms .................................................................. 5

Filipino American .....................................................................6

Pilipino versus Filipino...........................................................6

Midlife .................................................................................... 6

Health Belief Model .....................................................................6

Chapter 2

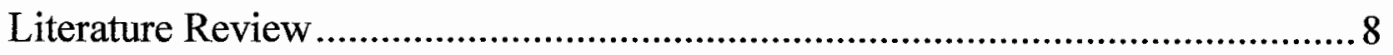

The Health Belief Model (HBM) ..................................................... 8

Common Health Concerns for Women in Midlife ................................. 10

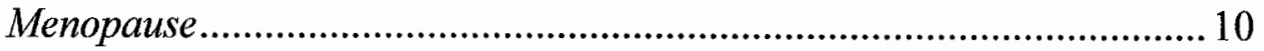

Type II Diabetes.................................................................... 11 


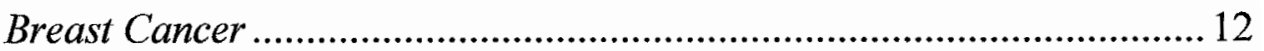

Cardiovascular Disease ........................................................... 12

Heart disease ...................................................................... 12

Hypertension ................................................................... 13

The Application of the Health Belief Model (HBM) .............................. 13

Visualizing its Use................................................................ 14

Filipino Immigrants Becoming Filipino Americans.............................. 16

A New Wave of Immigration......................................................... 16

Uncovering the Filipino Identity and Culture ................................... 16

Filipinos and the Concealing Effects of the Asian American Identity ........ 17

The Model Minority Smokescreen ................................................... 17

Other Concealing Factors ..................................................... 17

Language and Health Communication .......................................... 18

Health Care Experiences.......................................................... 19

Health care utilization............................................................. 19

Mental health counseling ....................................................... 19

Filipino American Women and Health ......................................... 20

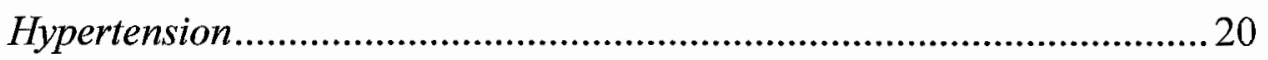

Breast Cancer ......................................................................... 21

Type II Diabetes ..................................................................... 21

The Health Belief Model among other Filipina Populations..................... 22

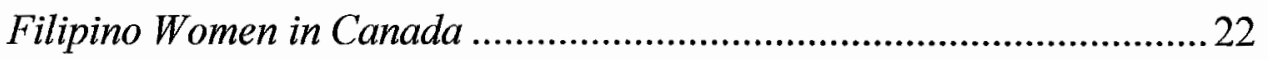




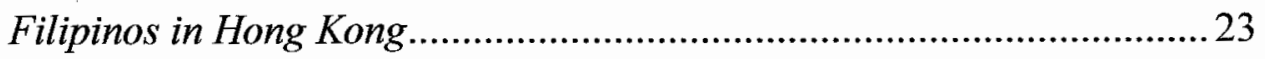

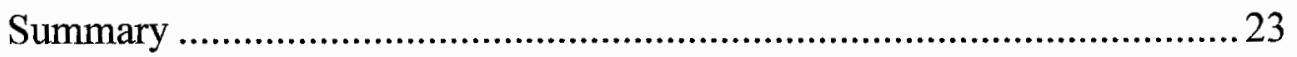

Chapter 3

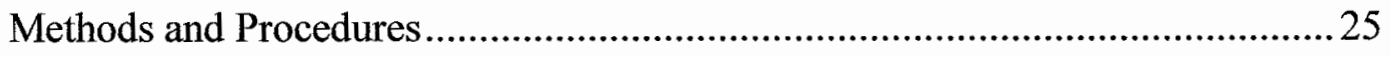

Research Design ......................................................................2 25

Sampling Procedures .............................................................. 26

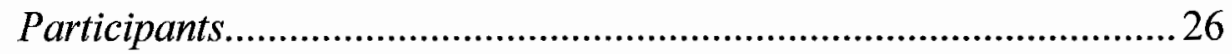

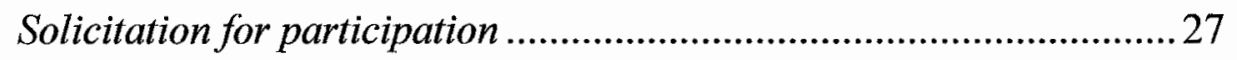

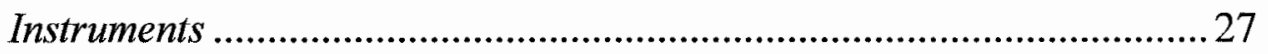

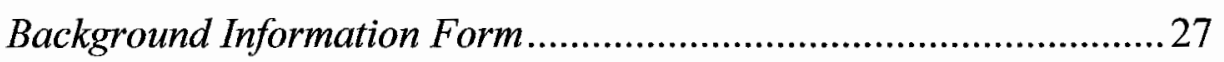

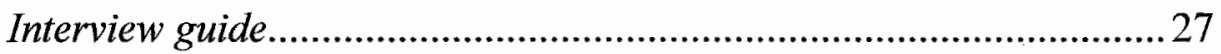

Procedure .......................................................................... 28

Face-to-face interview..................................................... 28

Member check meeting ..............................................................2 28

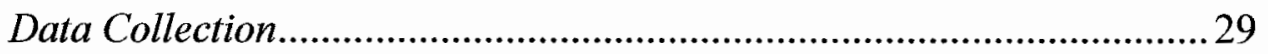

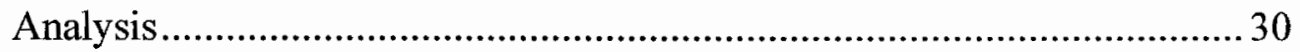

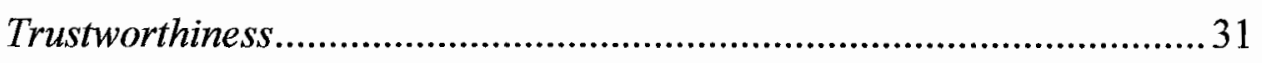

Triangulation ..................................................................... 32

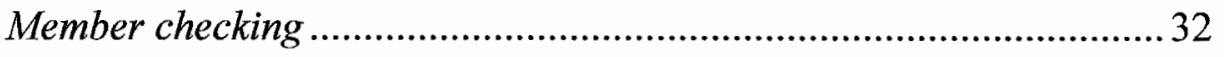

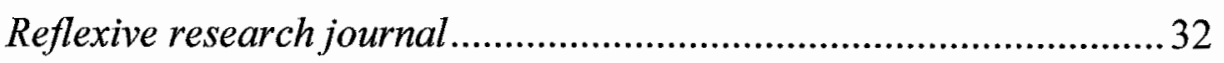

Ethical Treatment of Participants.................................................... 32

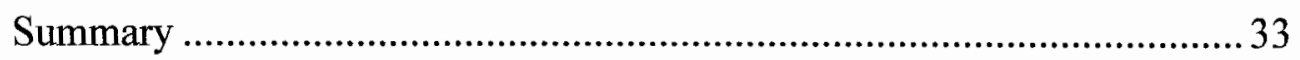

viii 


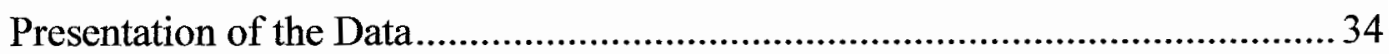

Description of the Participants ........................................................... 34

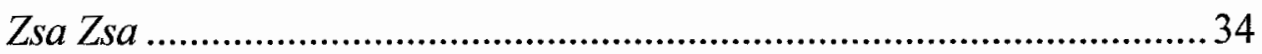

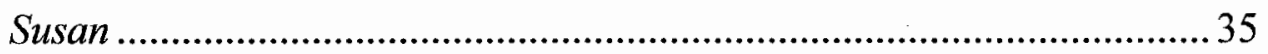

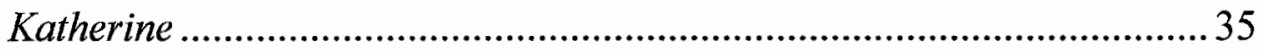

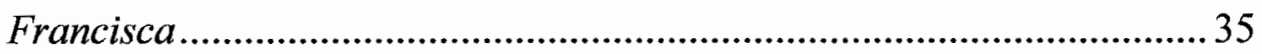

Presentation of Interview Responses .................................................... 36

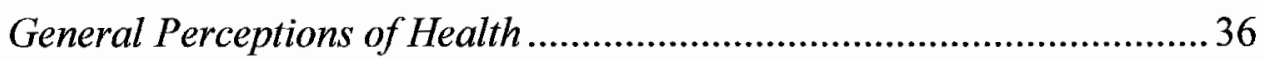

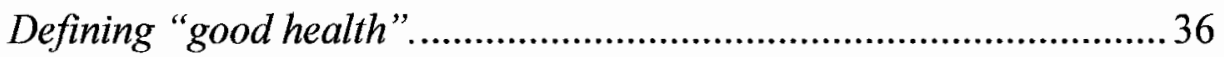

Recognizing changes in health ................................................ 37

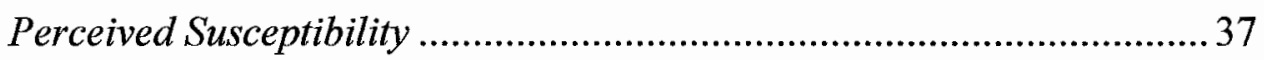

Susceptibility to a cardiovascular disease ...................................... 37

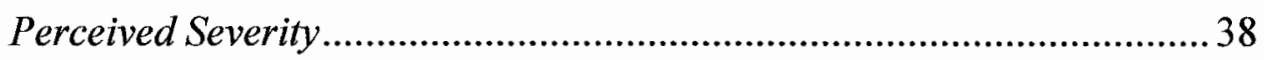

Severity regarding a cardiovascular disease ................................... 38

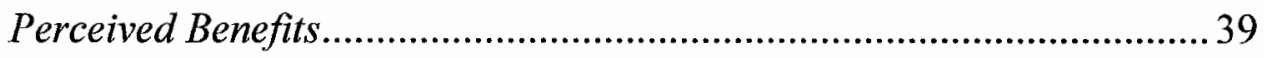

Flexibility of services and perceptions of the health care

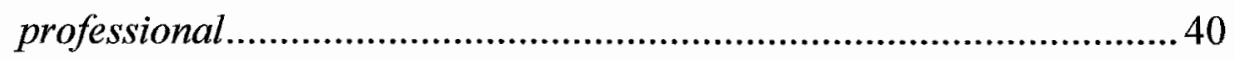

Perceptions of gender, ethnicity, and age .....................................4 41

Other positive perceptions of the health care services ......................41

Perceived Barriers ................................................................. 42

Non-Western medical perceptions ............................................42

Negative experiences with the health care services.........................4 43 
Cues to Action and Self-Efficacy ............................................................ 43

Finding and using resources for current health issues ......................... 44

First exposure to information about cardiovascular diseases .............. 44

Cues to seeking health care services.................................................... 45

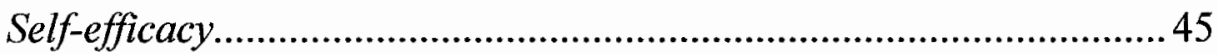

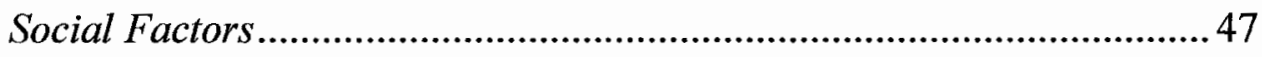

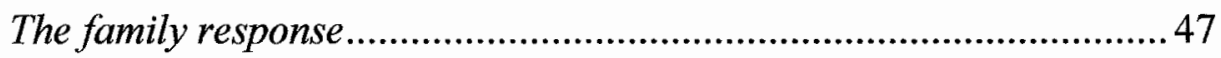

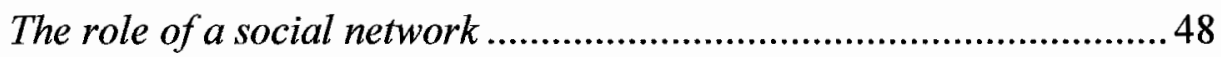

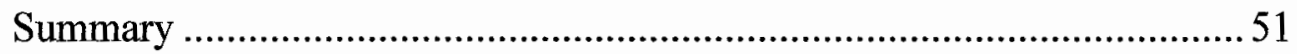

\section{Chapter 5}

Summary, Conclusions, and Recommendations ...............................................52

Summary of the Research Process............................................................. 52

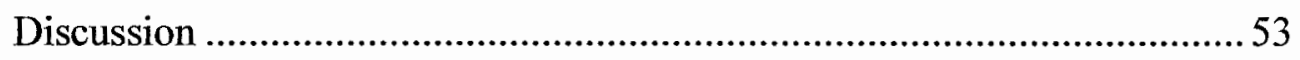

Basic Understanding of Health as a Midlife Filipino American

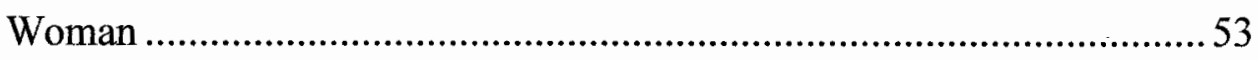

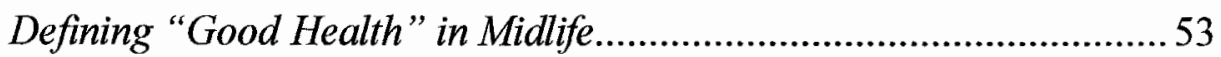

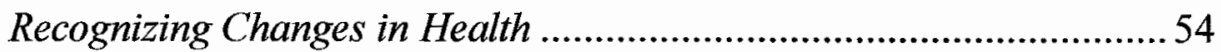

What do Midlife Filipino American Women Believe about their Risks

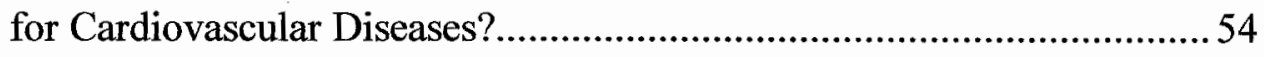

Perceived Susceptibility to a Cardiovascular Disease ..........................54

Perceived Severity of a Cardiovascular Disease ................................55 
What are their Beliefs in the Benefits of Utilizing Health Care

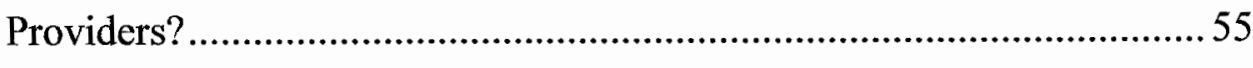

Flexibility of services and perceptions of the health care professional .. 55

Perceptions of Treatment Based on Gender, Ethnicity, and Age .......... 56

Other Positive Perceptions of the Health Care Services...................... 57

How do their Health Beliefs Affect Health Care Utilization?.................57

Cues to Action and Self-Efficacy ...............................................5 57

Finding and using resources for current health issues...................55

First exposure to information about cardiovascular diseases ..........58

Cues to seeking health care services ..........................................58

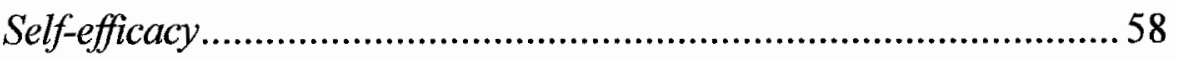

How does the Familial Unit Influence Attitudes Toward Health Care

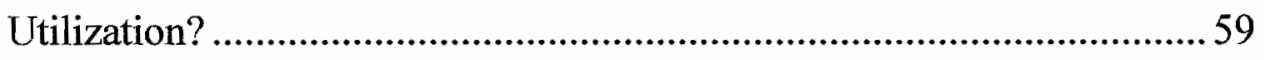

The Family Response ............................................................59

How does the Social Network Influence Attitudes Toward Health Care

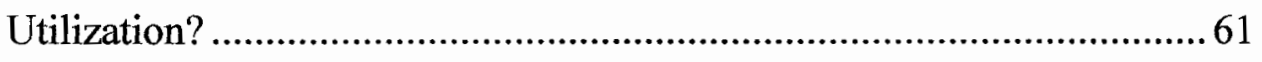

The Role of a Social Network .....................................................61

What are the Issues that Inhibit Midlife Filipino American Women from Confident Utilization of Medical Consultation and Available Health Care Resources in the US? ..............................................63

Perceptions of Non-Western Medicine ..........................................63 
Negative Experiences With the Health Care Services. 63

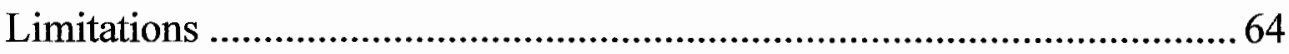

Sample Size and Proportion of Participants ..................................... 64

Established Criteria for Inclusion ...............................................64

Variety of Health Care Services and Providers .................................65

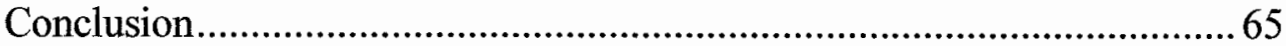

Recommendations and Suggestions for Future Research .......................6 68

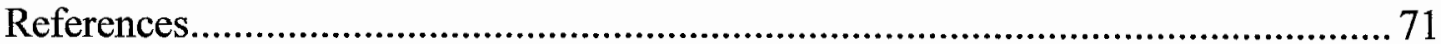

Appendix

A. Background Information Form ....................................................... 78

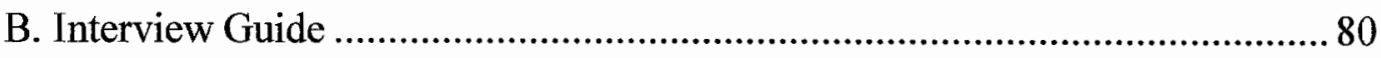

C. Informed Consent Form .................................................................. 83

D. Summary Outline of the Trends and Themes...........................................86 


\section{List of Figures}

Figure

2.1. Key concepts and definitions of the health belief model ............................

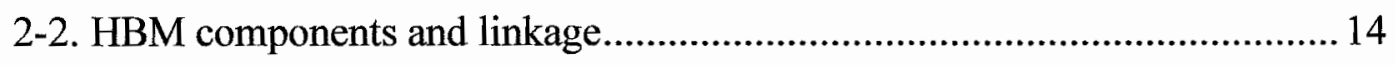

2.3. A conceptualization of the application of the HBM in assessing the health care utilization among midlife Filipino American women in regards to

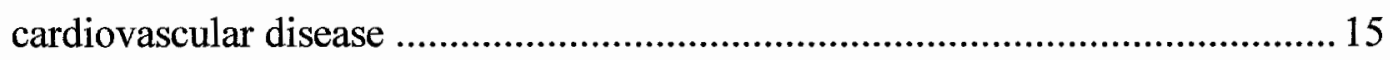




\section{Chapter 1}

Introduction

\section{Background of the Study}

In 1983, my grandmother died of a brain tumor at the age of 61 . Although there was nothing atypical about her death, she seemed relatively young to be inflicted with such a condition. What really startled me was my mother's age at the time: she was 26. When I turned 26 , the thought of losing my mother, who was just entering middle age, or midlife, was alarming, thus sparking an urgent curiosity in exploring the health concerns among midlife Filipino American women. I started asking my mother about her medical history while becoming increasingly concerned about her risk factors for certain health conditions. Yet in my attempts to find out which diseases and conditions for which she was at risk, I was shocked to find little information that directly addressed the health needs of midlife Filipino American women.

Filipino Americans constitute the second largest Asian American group in the United States (US Census Bureau, 2002), yet little is known about their health status. The greatest obstacle in finding research about Filipino Americans is that they are masked behind the term "Asian American", an extremely broad categorization of many cultures. Approximately 50 countries in the world are located in Asia and each country's population holds to its own customs (www.worldatlas.com, 2006). Despite the diversity among people of Asian descent who call the United States home, many North American-based researchers tend to clump the experiences of people from these various cultures into one group - namely Asian American. 
Research is surprisingly plentiful when Asian American populations are studied as one collective group, especially when addressing education in the United States. However, this "clumping" method also leads to misrepresentative experiences of specific Asian American populations. For example, the model minority label was pervasive in the educational literature and appears very specific to the Asian American population. Oyserman and Sakamoto (1997) found that Asian American students were aware of the rewards and the consequences of being labeled as a model minority. But this label may prove to be one of many layers that mask the health status of Asian Americans because of the implication that all Asian Americans and their experiences are similar when they, in fact, are not.

The greatest absence in Asian American research, in general, is that few researchers have focused on Asian American experiences in physical education, physical activity, exercise, and health in the United States. Of those few, they continued perspectives from an educational standpoint and discussed how the absence of Asian American physical educators and athletes, coupled with cultural expectations, negatively affected Asian American students' perceptions in pursuing a career in physical education, health, or sport (Shifflett, Buliavac, Howd, O'Brien, \& Seifert, 1991; Wong, 1999). This may be an additional barrier in obtaining accurate information about the health status of specific Asian American populations.

Nonetheless, contemporary medical advances and proactive attitudes toward health have resulted in increased longevity for people in the United States, including Asian Americans. The life expectancy of a female born after the 1940 s is over 70 years, more than 15 years longer than a woman born in the first half of the 20 th 
century (Arias, 2006). But even with this progress, there is no safeguard to suggest that every person, regardless of race or culture, is equally immune to disease.

\section{Statement of the Problem and Purpose}

Research pertaining to Filipino American health issues is growing but most remain delimited to children, college students, and older adults. The purpose of this study was two-fold. The first was to define the health beliefs of four Filipino American women who were in midlife through in-depth one-on-one interviews regarding cardiovascular diseases and in utilizing health care to prevent, diagnose, or treat those diseases. The second purpose was to conceptualize potential links between the health beliefs of those Filipino American women and their attitudes or choices about health care utilization.

\section{Justification}

From a broad health perspective, researchers know little about the health status of Asian Americans. They know even less of how each Asian American population addresses its health issues. While there are numerous online resources addressing health issues for specific Asian and Asian American groups, the limited empirical research available still relays the assumption that all Asian groups experience and deal with those issues in identical fashion. Additionally, researchers who have focused on specific Asian and Asian American groups have concentrated on certain age groups and genders but have neglected others, such as midlife women.

\section{Assumptions}

As the researcher, I based this study on the following assumptions. 
1. Midlife Filipino American women have few resources to verify the health problems for which they may be at risk given their age and ethnicity.

2. In general, Asian Americans, and Filipino American women in particular, are apprehensive about utilizing available health care resources.

\section{Delimitations}

I established the following parameters for the study.

1. All participants were women in their middle years, or midlife, (between 44-55 years old) at the time of the study.

2. Participants were born and lived in the Philippines for at least 14 years.

3. Participants immigrated to the United States and had earned naturalized citizenship.

4. Participants' parents were both Filipino.

\section{Limitations}

The following factors served as limitations to the study.

1. Because this was qualitative study, the sample size was small (Thomas, Nelson, \& Silverman, 2005), limiting the richness of experiences.

2. Only women in midlife (between $44-55$ years old) were sampled for this study.

3. The location of where the researcher conducted the study was not representative of all midlife Filipino American women.

4. The health care systems which the participants may or may not have utilized were not representative of all midlife Filipino American women. 


\section{Research Question}

The overall research question for this study was based on the health belief model. This question was: What are the health beliefs of midlife Filipino American women regarding cardiovascular diseases and how do those beliefs influence health care utilization?

I developed the following questions based on each specific construct of the health belief model as parameters to guide my inquiry in making discoveries addressing the overall question:

1. What do midlife Filipino American women believe about their risks for cardiovascular diseases?

2. What are their beliefs in the benefits of utilizing health care providers?

3. How do their health beliefs affect health care utilization?

4. How does the familial unit influence attitudes toward health care utilization?

5. How does the social network influence attitudes toward health care utilization?

6. What are the issues that inhibit midlife Filipino American women from confident utilization of medical consultation and available health care resources in the United States?

\section{Definition of Terms}

Health. The World Health Organization defined health as "a state of complete physical, mental and social well-being and not merely the absence of disease or infirmity" (2006). For the application of this study, I focused on the physical aspect of 
health with an emphasis on the self-perceived attitudes and beliefs toward cardiovascular health.

Filipino American. Throughout this text, I used the terms Filipinos and Filipino Americans to indicate and distinguish the Filipino people as a whole from other Asian subgroups. A majority of the literature I used for this study did not appear to indicate any stark differences between Filipino and Filipino American. Since researchers sampled Filipino American participants, I maintained consistency accordingly with authors in their use between these two terms.

Pilipino versus Filipino. There is an ongoing debate in the use of "P" versus "F". Historically, the sound of an "F" and the letter "F" itself did not exist in the predominant language, Tagalog (Nadel, 2004). Some researchers use Filipino and Pilipino interchangeably in their studies while others stay consistent with one or the other. Faustino (2003) stated that Pilipino was more descriptive of recent immigrants. Revilla (1997) reiterated the conflict that some Filipinos view the pronunciation of the "P" as derogatory while others use it as a means of preserving and promoting their heritage. For the application of this study, I used the term Filipino consistently as it was also representative of current research.

Midlife. Midlife, or middle age, is defined as "the period of human life between youth and old age, sometimes considered as the years between 45 and 65 or thereabout" (dictionary.reference.com, 2007). For the application of this study, I adjusted this range to $44-55$ years old.

Health Belief Model. The health belief model (HBM) is a value expectancy theory “...designed to exclusively predict health behaviors based on the constructs of 
perceived susceptibility, perceived severity, perceived benefits, perceived costs, cues to action, and self-efficacy" (Sharma \& Romas, 2008, p.255). For this study, I used "barriers" in place of "cost" as that term is more commonly used in the health literature. 
Chapter 2

\section{Literature Review}

The purpose of this study was two-fold. The first was to define the health beliefs of four Filipino American women who are in midlife through in-depth one-on-one interviews regarding to cardiovascular diseases and in utilizing health care about those diseases. The second purpose was to conceptualize potential links between the health beliefs of those Filipino American women and their attitudes or choices about health care utilization.

There are three major sections in this literature review. In the first section, I provide an overview regarding the development and theoretical constructs of the health belief model. I also discuss some of the common health concerns for women in midlife. In the second section, I center on the Filipino American experiences, first as an Asian immigrant population, and eventually as an underrepresented population in health literature. For the final section of the chapter, I provide a brief synopsis of the health care experiences and perceptions of Filipino women who reside in other countries.

The Health Belief Model (HBM)

Researchers developed the health belief model (HBM) in the $1950 \mathrm{~s}$ in order "...to explain the widespread failure of people to participate in programs to prevent and detect disease" (Janz, Champion, \& Strecher, 2002, p.46). At the time, public health officials were puzzled by the low patient turnout to free health screening programs. In turn, they recruited social psychologists to examine what was preventing its utilization and eventually the beginnings of the HBM emerged (Janz, et al., 2002). 
There are six constructs of the HBM: perceived susceptibility, perceived severity, perceived benefits, perceived barriers, perceived benefits, cues to action, and selfefficacy. Each of these constructs is defined in Figure 2.1.

\begin{tabular}{|l|l|l|}
$\begin{array}{l}\text { Concept } \\
\text { Perceived } \\
\text { susceptibility }\end{array}$ & $\begin{array}{l}\text { Definition } \\
\text { One's belief regarding } \\
\text { the chance of getting a } \\
\text { condition }\end{array}$ & $\begin{array}{l}\text { Application } \\
\text { Define population at risk, risk levels; } \\
\text { personalize risk based on a person's } \\
\text { characteristics or behaviors; make } \\
\text { perceived susceptibility more } \\
\text { consistent with an individual's actual } \\
\text { risk }\end{array}$ \\
\hline $\begin{array}{l}\text { Perceived } \\
\text { severity }\end{array}$ & $\begin{array}{l}\text { One's belief of how } \\
\text { serious a condition and } \\
\text { its sequelae are }\end{array}$ & $\begin{array}{l}\text { Specify consequences of the risk and } \\
\text { the conditions }\end{array}$ \\
\hline $\begin{array}{l}\text { Perceived } \\
\text { benefits }\end{array}$ & $\begin{array}{l}\text { One's belief in the } \\
\text { efficacy of the advised } \\
\text { action to reduce the risk } \\
\text { or seriousness of impact }\end{array}$ & $\begin{array}{l}\text { Define action to take: how, where, } \\
\text { when; clarify the positive effects to be }\end{array}$ \\
\hline $\begin{array}{l}\text { Perceived } \\
\text { barriers }\end{array}$ & $\begin{array}{l}\text { One's belief about the } \\
\text { tangible and } \\
\text { psychological costs of } \\
\text { the advised action }\end{array}$ & $\begin{array}{l}\text { Identify and reduce perceived barriers } \\
\text { through reassurance, correction of } \\
\text { misinformation, incentives, assistance }\end{array}$ \\
\hline Cues to action & $\begin{array}{l}\text { Strategies to activate } \\
\text { one's "readiness" }\end{array}$ & $\begin{array}{l}\text { Provide how-to info, promote } \\
\text { awareness, employ reminder systems }\end{array}$ \\
\hline Self-efficacy & $\begin{array}{l}\text { One's confidence in } \\
\text { one's ability to take } \\
\text { action }\end{array}$ & $\begin{array}{l}\text { Provide training, guidance in } \\
\text { performing action; use progressive goal } \\
\text { setting; give verbal reinforcement; } \\
\text { reduce anxiety; demonstrate desired } \\
\text { behavior }\end{array}$ \\
\hline
\end{tabular}

Figure 2.1. Key concepts and definitions of the health belief model (Janz, et al., 2002, p.49)

In the following section, I discuss a few health issues and illnesses relating to midlife women and provide some background information on each condition. Later on in the chapter, I provide an example how a health care specialist could apply the health belief model to determine a perspective about a specific condition that would then allow for the development of strategies for behavior change or modification. 


\section{Common Health Concerns for Women in Midlife}

Women in midlife encounter a unique set of health concerns that they generally do not experience while in their 20s, 30s, and even their early 40s (Reichman, 1996). They may also face issues that affect women of all ages in a different manner. While there are many possible health concerns, the intent of this section is to highlight a few of the major issues and how they affect midlife women.

\section{Menopause}

Menopause is an event exclusive to women in midlife. Shimp and Smith (2000) defined menopause as "the final menstrual period" (p.93). In contrast to the other health issues, menopause is not a disease but rather an indicator of change (Porchino, 1983). Just as every female eventually starts her reproductive cycle, so too does it end. The average age of the onset of menopause is 51 , with $95 \%$ of females between 44-55 years old experiencing the actual process (Shimp \& Smith, 2000). The consequences of menopause are a major concern for midlife women primarily because of the decreased production of estrogen. Lower hormone levels increase the risk of osteoporosis and cardiovascular diseases (Shimp \& Smith, 2000).

\section{Osteoporosis}

For some midlife women, the termination of the menstrual cycle leaves them in a liberated yet vulnerable situation. One of the diseases for which they become increasingly at risk for is osteoporosis. Kaplan-Machlis and Bors (2000) define osteoporosis as "a universal, gradual reduction in bone mass to a point where the skeleton is compromised, resulting in fractures from minimal trauma" (p.632). Both men and women are susceptible to osteoporosis but menopausal women greatly 
increase their risks because of the change in hormonal levels, especially decreased estrogen production (Porchino, 1983). This disease is a major public health concern because decreased bone composition increases the likelihood of fractures, especially with injuries that affect mobility (Cauley \& Danielson, 2000).

\section{Obesity}

Obesity is a descriptive term for excess body fat (Flegal, 2000). Many current researchers are focusing their efforts on the growing epidemic in children but obesity remains a major concern for all age groups and genders (Harper, 2006). This condition is also a major health concern because carrying excess body fat increases a person's risks for various diseases, particularly cardiovascular diseases and diabetes (Flegal, 2000).

\section{Type II Diabetes}

Body weight conditions such as obesity are risk factors for diabetes and other ailments. In turn, diabetes is a strong risk factor for coronary heart disease in women (Newton, Lacroix, \& Buist, 2000). Lotufo, Sabolsi, and Manson (2000) noted that incidence rates of diabetes are generally higher in women than in men but they did not mention age differences at the onset between genders. There are two common forms of diabetes: Type I is a condition where there is a lack of insulin hormone production; Type II, also called adult-onset diabetes, is more common and is the condition wherein insulin is available but cells in the body are not using it efficiently (National Institutes of Health, 2007). 


\section{Breast Cancer}

Cancer is "...the leading cause of death among women aged 34-74" (Devesa, 2000, p.863) and the second leading cause of death among women of all ages. While the manifestation of cancer in the body varies by gender, breast cancer is the most common form of cancer among women (Ursin, Spicer, \& Bernstein, 2000). Some associated risk factors include obesity and increased age (Reichman, 1996). Midlife women are especially at risk due to menopause. Ursin, et al., (2000) found that breast cancers risks are as much as two times greater in women who experienced menopause in their 50s than women who experienced menopause while they were in their 40s.

\section{Cardiovascular Diseases}

Cardiovascular diseases envelop a myriad of health ailments specific to cardiac health, including heart disease, stroke, atherosclerosis, and artery disease. I chose to focus on two particular illnesses, heart disease and hypertension, based on emergent statistics that these two conditions are more prevalent within the Filipino American population (Filipinos in the United States, 2003).

Heart disease. Heart disease, more commonly known as coronary heart disease, is a general classification for any disorder that hinders the normal functions of the heart (Holubkov \& Reis, 2000). Coronary heart disease is a condition where the build up of plaque in the artery walls of the heart limits the flow of oxygen-rich blood needed for the heart to function (Center for Disease Control, 2006). No longer exclusively a "man's disease", heart disease is the leading cause of death in women (Ness, 2000; Newton, et al., 2000). 
Hypertension. Hypertension is a major risk factor for cardiovascular disease and heart disease. Commonly known as high blood pressure, hypertension is a condition where blood pressure is abnormally high (e.g. $140 / 90 \mathrm{~mm} \mathrm{Hg}$ ). It is an especially important concern among the midlife female population because of the general trend in weight gain during that period of time, regardless of ethnicity (Brown, Matthews, Bromberger, \& Chang, 2006). Uncontrolled hypertension is also a risk factor for myocardial infarction (MI) or a heart attack. Myocardial infarction is a major health threat, accounting for over $50 \%$ of sudden deaths in women (Reichman, 1996).

The Application of the Health Belief Model (HBM)

The intent of this study was to explore beliefs within a specific population by applying constructs of the HBM in regards to cardiovascular disease and health care utilization. In summary, health promotion specialists and other health professionals use the HBM as a framework to identify strategies for effective change when a person has already constructed their own views of and placed their own values and preconceived outcomes on a desired behavioral change.

The following figures are visual representations of the HBM. Figure 2.2 is a visual "flowchart" representation of the constructs of the HBM and how they are linked together to determine an effective behavior change. In Figure 2.3, I provide a visual conceptualization of predicted beliefs from which I could utilize the constructs of the HBM in assessing the health care utilization among midlife Filipino American women in regards to cardiovascular diseases. 
Visualizing Its Use

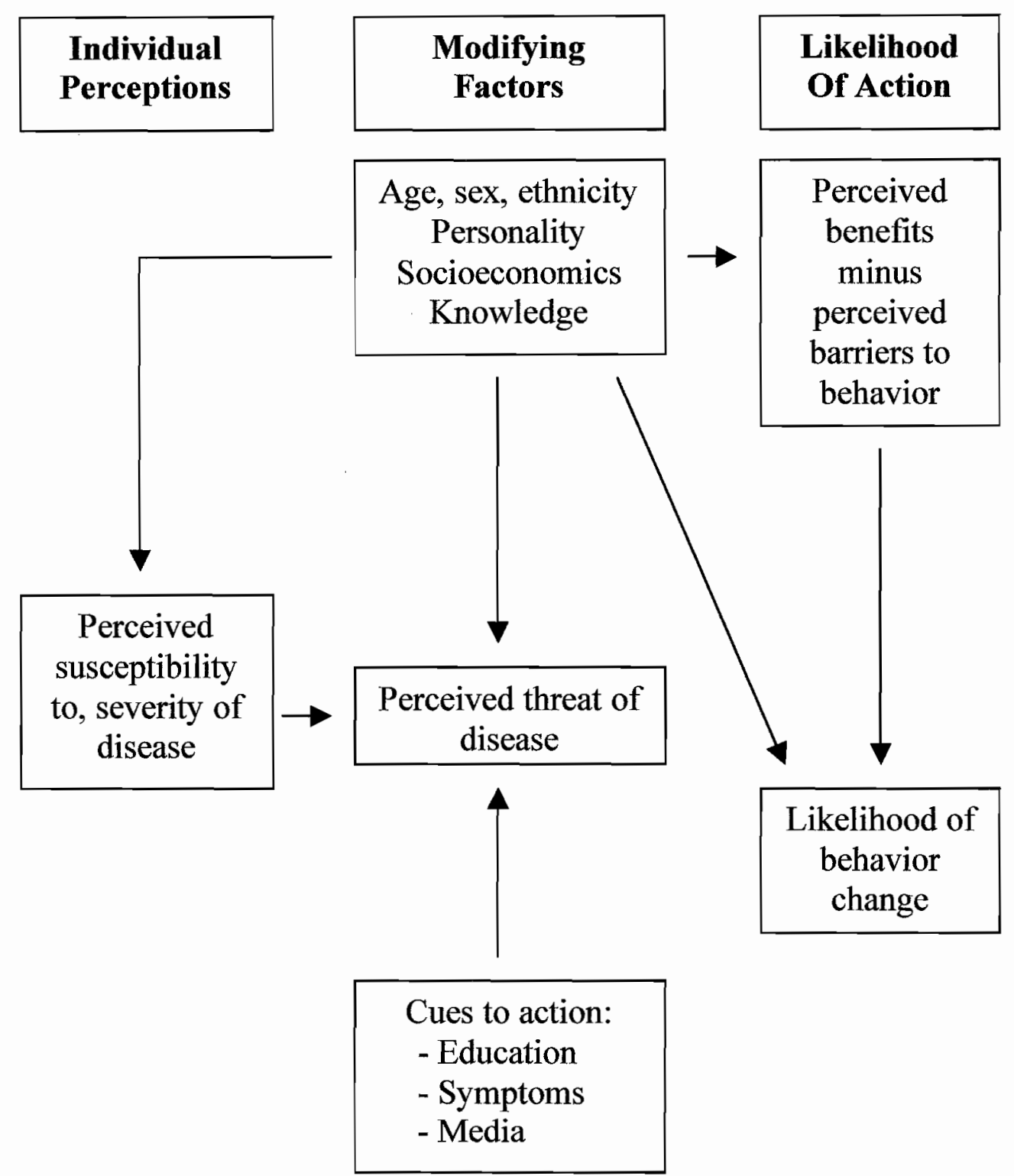

Figure 2-2. HBM components and linkage (Janz, et al., 2002, p.52) 


\section{Conceptualizing the Application of the Health Belief Model}

Target Population: Midlife Filipino American women

Problem: Under-utilization of health care resources because personal beliefs in seeking information about certain health issues through providers are culturally hindered Behavior/action plan: Become a more proactive patient in finding out how personal lifestyle and culture affect risks associated with CV diseases; do a litle research on own time, then bring further questions to health care professional

Construct

Perceived Susceptibility: I could develop CV diseases

Perceived Severity: I will not be able to perform my duties as a woman, wife, mother or may die much sooner

Perceived Bamiers: I'm too young to develop CV disease; I have done enough for my health to reduce the chances; 1 have no idea how to tell if $I m$ at risk; $I$ don't like using the health care sources that are available to me

Perceived Benefits. I will be able to learn more about CV health prevention; 1 will understand how to modify my lifestyle to decrease my risks for illness or learn to cope

Cues to Action: I was surprised that my spouse was diagnosed with a CV illness, we thought we were too young and too good in shape

Self-efficacy issues: I don't know how to talk to my health care professional because he/she doesn't understand how my cultural background influences my decisions
Application

Reaffirm threat of disease but reassure that seeking information may ease doubts or uncertainties Reinforce factual information retrieved from medical professionals that developing a disease does not always greatly affect lifestyle and duties; encourage utilizing peers, friends, and family members to assist in duties

Provide information supported by medical and health professionals on how lifestyle choices may influence risks of developing CV diseases; begin to advocate use of health care resources in finding info relevant to personal concerns

Being a better informed patient will allow higher self-confidence and willingness to ask more questions, seek multiple perspectives from health care resources if possible

Think about the habits that can be changed or modified; spouse, very possibly a male, may have differing risk factors but personal habits may need to be reevaluated

Seek a trusted friend or relative to accompany you for moral support, research on own time the risks associated with particular disease, ask questions relating to personal lifestyle risk factors; seek another professional, if possible

Figure 2.3. A conceptualization of the application of the HBM in assessing the health care utilization among midlife Filipino American women in regards to cardiovascular disease 
Filipino Immigrants Becoming Filipino Americans

Filipino Americans are the second largest Asian American group in the United States (US Census Bureau, 2002). Yet despite their prominence, several factors contribute to the problem of their under-representation in health-based literature. A starting point in determining an answer may be to figure who they are.

\section{A New Wave of Immigration}

Filipinos immigrated to the United States in large numbers following World War II and after the enactment of the Immigration Act of 1965 (Allen, 1977). In contrast

to "immigrant waves" of male Filipino laborers in the early $20^{\text {th }}$ century, more Filipinos began moving to the States as family units (Litton, 1999). Many were already highly educated professionals such as doctors, nurses, and engineers, entering with the hopes of attaining spiritual and economic prosperity (Faustino, 2003).

\section{Uncovering the Filipino Identity and Culture}

Filipinos fall into a unique yet perplexing identity gap. Although they are geographically situated near the Asian continent, they hail from a cultural background that is a stark contrast to typified Asian cultures. Because the Philippines endured a 400-year era of Spanish rule, the Filipino identity was heavily influenced by Western culture (Faustino, 2003). During the United States occupation of the Islands in the early $1900 \mathrm{~s}$, American teachers furthered Western ideals, giving instruction to Filipino students in English and presenting American values in the schools (Pido, 1997). The misperception that Filipino immigrants arrived in the United States "well assimilated" into American culture left them in a gap, mainly that they did not to be 
looked after or need additional assistance adjusting to Western culture. This issue was compounded by the notion that Filipinos also could not identify or sympathize with their Asian neighbors (Cimmarusti, 1996). Cimmarusti (1996) best described them as an invisible Asian group.

Filipinos and the Concealing Effects of the Asian American Identity

\section{The Model Minority Smokescreen}

Asian Americans are a mix of many ethnic minority groups in the United States. Yet they often automatically fall into a generalized image as the model minority, especially in the United States. The media, educators, employers and even Asian Americans themselves employ different types of reinforcement to uphold a particular standard to which all other minority populations are compared to (Ho, 2003). However, that model minority measuring stick is problematic.

In the academic setting, the model minority label is pervasive but it may also be likely that educators are unknowingly using it. The simplistic viewpoint that a student of Asian descent is hard-working, obedient, and concentrated on performing his or her best in the classroom (Wong, 1980), masks the complex issues an Asian American student might be facing in order to attain that status. The perception of a person who does his or her duties as told without protest may be a barrier in assessing the health status of Asian Americans in the sense that an Asian American will not admit to being in poor health when he or she is.

Other Concealing Factors. Gaining access to Asian American females to assess their health status is particularly challenging due to a myriad of reasons, some specific to each culture and others that encompass the female role in Western society. 
Berg (1999) described a vast array of barriers including language differences, an inability to trust researchers, and the restrictions some females face based on a patriarchal society. Other reasons included juggling the demands of home and work; in essence, time is valuable, although this aspect may be typical of women regardless of ethnicity. Language discrepancies among Asian American populations and their health care resources appear to be the biggest obstacle.

\section{Language and Health Communication}

Some people have a difficult time understanding their health professionals, even when their primary language is English (Ngo-Metzger, Legedza, \& Phillips, 2004). Asian Americans again appear to lose ground in this case but the level of miscommunication, possibly the inability to communicate at all, also depends on the Asian American group. For example, the Chinese do not have a descriptive word for Alzheimer's disease (Srinivasa \& Guillermo, 2000). As a result, some Asian American groups ultimately have a difficult time understanding the severity of diseases because they have no conceptualization of what they are.

Equally detrimental to misunderstanding the medical language is the fact that some Asian populations are unable to convey their health status because they speak one language at home and another language elsewhere. Yu, Huang, Schwalberg, Overpeck, and Kogan (2002) examined the adverse effects Asian American students experienced because they were caught between two different languages. While their parents spoke only in their native tongue at home, the students were learning about health issues at school in English. The underlying issue was that they could not explain their problems to their parents because of the language disparity. Children 
who came from households where the primary spoken language was not English were at a higher risk for health problems, especially feelings of isolation and other psychological problems (Yu, et al., 2002).

Health Care Experiences

Asian Americans were less likely to report positive experiences with their doctors than White Americans (Ngo-Metzger, et al., 2004). They reported garnering less attention, direction, and useful counseling from their doctors. Part of this disparity might be due to the doctor's lack of knowledge in how to inform such patients of health problems. The model minority characterization plays a role in how doctors interact with patients. There may be an assumption that a patient's work and lifestyle ethic provide a buffer against disease (Ngo-Metzger, et al., 2004).

Health care utilization. For some patients, their health care experiences with one provider are so dissatisfying that they choose to switch to another. Kennedy, Taylor, and Lee (2005) found that women changed providers for two main reasons: 1) they left because of the provider's perceived inability to communicate or listen effectively and 2) they wanted a provider who can relate culturally and by gender. Although Asian American women were not sampled in this particular article, my assumption is that it is possible that they share the same attitudes toward their own health care providers.

Mental health counseling. Cimmarusti (1996) specifically explored FilipinoAmerican perceptions on familial responsibilities and expectations and how these impacted mental health. Much of the tradition of collectivistic expectations (e.g. putting the family first, paternalistic) associated with Asian families reemerged. 
However, the author made some bold recommendations in the way health professionals should approach Filipino Americans about their health issues. There appeared to be a "sensitivity" marker wherein a health specialist may expect either an entire family to fully embrace therapy as well as the therapist or garner a complete "shut-out" by the family. Chun and Sue (1998) reiterated how some Asian Americans use their health care system "only after [their] mental health has seriously deteriorated" (p.76).

Filipino American Women and Health

Research pertaining to Filipino American health issues is growing but most remains delimited to children, college students, and older adults. The intent of this section was to illustrate how visible (or invisible) Filipino American women are in health literature.

\section{Hypertension}

Yee (1997) found that high blood pressure is a significant public health problem among Filipinos. High blood pressure, also called hypertension, is a major concern because it is a risk factor for heart disease, the leading cause of death among Filipino Americans (Filipinos in the United States, 2003). Yet despite research stating that hypertension rates among Filipino Americans are increasing (Brown, et al., 2006) they are still largely excluded from cross-cultural studies. For example, Brown et al., (2006) examined if there was a positive correlation between perceived unfair treatment and elevated blood pressure. Although there was no correlation, African American, Chinese, and Japanese women expressed unfair treatment as a result of 
their race, while White women viewed the discrimination based on their gender. It is unknown how Filipino American women view this problem.

\section{Breast Cancer}

Breast cancer is the number one killer among Asian American women yet many avoid utilizing cancer screening methods or cancer treatments (Wu \& Bancroft, 2006). Gelber, McCarthy, Davis, and Seto, (2006) examined disparities in breast cancer management within the Asian American and Pacific Islander (AAPI) populations, including Filipino women. They did not look specifically at how an individual health care professional responds to an AAPI patient but their findings did reveal differences in accepting treatment. Japanese and Filipino women were less likely to undergo breast-conserving surgery (BCS) and subsequent radiation therapy following BCS as compared to White women. However, the researchers did not explain why the women in the study chose not to resort to those procedures. It is possible that this avoidance could be attributed to cultural differences between the health care professional and the patient (Wu \& Bancroft, 2006).

\section{Type II Diabetes}

Yee.(1997) noted that research linking Asian Americans to diabetes is scarce. Recently however, researchers have discovered that Filipinos had a higher incidence rate of diabetes than previously thought (Gomez, Kelsey, Glaser, Lee, \& Sidney, 2004). Araneta and Barrett-Connor (2005) further revealed how standardized measuring tools such as the Body Mass Index (BMI) misdiagnose Asian and other non-White populations in regards to risk factors associated with excess body weight, such as developing diabetes. Using a sample of Filipino, African American, and 
White women, Araneta and Barrett-Connor (2005) illustrated how visceral adipose tissue (VAT) differed between populations. Extensive use of the BMI scale did not take into account VAT levels even though "[e]xcess VAT is a known risk factor for type II diabetes" (p.1463).

The Health Belief Model among other Filipina Populations

Filipino American women are not the only ones residing in Western society. The following two summaries illustrate how researchers have addressed health beliefs among Filipino women living in other countries. Even here it is apparent that Filipino women, regardless of their residence status, are still highly underrepresented in health literature.

\section{Filipino Women in Canada}

Farrales and Chapman (1999) explored the beliefs about food, body, and health within a group of Filipino women residing in Canada. Not surprisingly, Canadians subscribe to views regarding desirable body size, negative attitudes about food and eating, and factors contributing to disease risk in similar fashion to US perspectives. The researchers found that this group of Filipino women had conflicting beliefs between their own heritage and their adopted one. For example, thinness in females is a desired characteristic in the United States and Canada, yet to some Filipinos, thinness exudes malnourishment and poor health (Farrales \& Chapman, 1999). In regards to disease prevention, the Filipino women expressed contradictory views and attitudes about being underweight. Rather than subscribing to a thin body frame, "fatness" was a common theme, signifying that carrying a little more weight is advantageous in disease resistance. 
Filipinos in Hong Kong

Sometimes, upheld beliefs about a system prevent its utilization. Barriers contributing to this resistance are often lack of knowledge about the system or the belief that there is no need to use it (Ford, 1997). Filipino women in Hong Kong utilized cervical cancer screening resources only if they were previously screened. Interestingly over three-quarters of the sampled population knew of cervical cancer screening methods yet more than half were not utilizing those resources. Immigrant women in general underutilize health care and will seek help in a health care system only when all other methods fail (Holroyd, Taylor-Piliae, \& Twinn, 2003). The researchers suggested that doctors and nurses should ideally culturally match their patients for greater success in advocating screening usage.

\section{Summary}

The HBM provides an exceptional application in understanding the opinions and perceptions of sub-populations otherwise hidden because of generalizations about the larger population. Much of the research in this chapter illustrated how those generalizations essentially invalidate or make invisible the existence of a very diverse set of people.

The most apparent discrepancy throughout the review was how invisible Filipino American women or, for that matter, Filipino Americans in general, are in health literature. It is possible that Filipino American women are not regularly sampled for studies because there was enough representation through more traditional views of who Asian women are and what it defined as being an Asian woman. Two underlying themes compounding the problem in determining the health status of Filipino 
Americans also emerged: 1) Filipinos are considered an Asian group so they are part of the model minority and are not expected to seek out help. This may be in part because of their self-sufficiency as perceived by American society and medical professionals; and 2) Filipinos supposedly understand American expectations and culture because they were educated by Americans so they do not need further assistance in transitioning and immigrating to the United States. 


\section{Chapter 3}

Methods and Procedures

The purpose of this study was two-fold. The first was to define the health beliefs of four Filipino American women who were in midlife through in-depth one-on-one interviews regarding cardiovascular diseases and in utilizing health care to prevent, diagnose, or treat those diseases. The second purpose was to conceptualize potential links between the health beliefs of those Filipino American women and their attitudes or choices about health care utilization.

This chapter contains information on the research design, the sampling procedures, including the profile and recruitment procedures of potential participants, and the methodological procedures. It also includes further details about the background information form, the development of the interview guide, the procedures for data collection and analysis, and the ethical treatment of participants.

\section{Research Design}

This research was a qualitative case-study of midlife Filipino American women's health beliefs. Marshall and Rossman (2006) describe qualitative research as "...a broad approach to a study of social phenomena" (p.2). As such, it takes on many facets that emerge rather than ones which are predetermined (Creswell, 2003). It is also interpretive (Marshall \& Rossman, 2006). For example, unlike data obtained in a quantitative design, I extracted data that cannot be statistically interpreted. The data for this study came from in-depth interviews with four female Filipino immigrants in midlife (between 44-55 years old) who became American citizens, and who reside in Central California. 
In qualitative research, the researcher is not just a spectator. Rather he or she is more of an interactive tool for data collection and analysis (Marshall \& Rossman, 2006). I employed a qualitative case design for the following reasons: First, I wanted to understand why the status of Filipino American health was difficult to find in the current health literature. Second, most studies regarding Asian Americans and their health were limited to quantitative data, an approach which shed light on the health concerns they knew about in general but provided little to no differentiation in how each subpopulation, such as Filipino Americans, responded to those concerns. Finally, I wanted to discover any reasons why Filipino Americans would seek or refuse health care. Researchers suggested that some Asian American populations did not seek out health care resources based on a myriad of culturally influenced reasons. Sampling Procedures

Participants. Because the nature of this study required face-to-face interaction, I purposefully chose the participants: Midlife Filipino women who were affiliated with a local Filipino American club (Creswell, 2003; Thomas, Nelson, \& Silverman, 2005). I chose this particular group primarily because of access to potential participants as well as the nature of the club's mission in promoting and preserving Filipino culture.

In a quantitative approach, a larger sample size strengthens the validity of the instruments and analysis used for the study. In contrast, a qualitative approach is enhanced when research "...stresses the importance of context, setting, and participants' frames of reference" (Marshall \& Rossman, 2006, p.54). Qualitative studies, then, work best using a small sample group (Thomas, et al., 2005). A total of 
four participants who met the criteria for inclusion were individually interviewed in March, April, and October of 2007.

Solicitation for participation. I contacted a female member of the Filipino club who offered the names and phone numbers of six other female members who met the age criteria for inclusion. The woman who gave me the information was not included in the study as a control for confidentiality purposes. I contacted all six participants and conducted a short phone interview by administering the background information form. Four participants were chosen based on the delimitations established for this study and on their availability for an interview.

\section{Instruments}

Background Information Form. I designed a background information form in order to screen potential participants. Based on the delimitations for this study, the questions on the form included age, citizenship status, information regarding the participant's family, and health care status (see Appendix A).

Interview guide. Patton (2002) defined an interview guide as "...questions or issues that are to be explored in the course of an interview" (p.343). To some degree, an interview guide is an outline of topics to discuss, some of which may not be addressed at all and others which in further inquiry and in-depth information will provide a clearer conceptualization of the issue at hand (Patton, 2002). I based the primary categories of the interview guide on this study's research questions. Appendix B contains a detailed copy of the interview guide. 


\section{Procedure}

I began interviews with participants in mid-March 2007 and completed data collection and transcription in October 2007. I used the following methods to obtain data: face-to-face interviews, member check meetings, and a reflexive research journal.

Face-to-face interview. Marshall and Rossman (2006) suggested that an interview is just one of many methods used in a case study approach. I chose to conduct interviews because of its value in emphasizing "...close, personal interactions between researcher and participant..." (p.56). The justification was further enhanced by the notion that "....intense interactions provide[s] subjective understandings that can greatly increase the quality of qualitative data" (Toma, 2000, as cited in Marshall \& Rossman, 2006, p.62).

I conducted a pre-briefing session at the beginning of the interview, which included an informed consent form (see Appendix C). The focus of the pre-briefing session before the first interview was to review the purpose the study, the right to ask questions, and the right to express comments that were not to be included in the study. Once the participant agreed to the terms, I interviewed her using a digital recorder, as well as taking notes on a printed copy of the interview guide. Each interview lasted between one and two hours.

Member check meeting. The purpose of the member check meeting was for clarification and accuracy purposes of the participant's responses (Punch, 1998) and a chance to voice any other comments or concerns she may have had. Once the face-toface interview was completed, I de-briefed the participant by informing her that I 
would check back for a follow-up interview, or member check meeting, once her interview data were transcribed and reviewed.

Although I had proposed two individual meetings, a face-to-face interview and a member check meeting, I only fulfilled the face-to-face meeting with each participant. I conducted a member check via telephone and email to make sure the participants had the opportunity to address any questions or inquiries after they received a copy of their respective interview transcript and a copy of Chapter 4.

\section{Data Collection}

This was a qualitative study in which data were in the form of digitally-recorded verbal responses garnered from face-to-face interviews. As such, the primary means of data analysis involved transcribing and documenting responses. Since there was a possibility of errors in auditory transcription and transfer to an electronic word processing program, I utilized member checks with each participant to confirm that the data were representative of what she said.

Due to time constraints, I followed up with each participant via electronic mail or by telephone inquiries in order to obtain consent that their respective responses were adequate. I was able to communicate with one participant via email who stated that she did not have any questions or concerns after reviewing her transcripts. I enlisted the assistance of another Filipino club member to return the transcripts by hand to the remaining participants since she had easier access to them. I left telephone messages with the three participants with instructions to review their transcripts and subsequently made contact with all of them by the end of February 2008. Since the three remaining participants each stated that they were satisfied with how I 
represented their responses and that the telephone conversations were no more than five minutes in length, I did not record those conversations; I made references to those conversations in my reflexive research journal.

\section{Analysis}

My inspiration for this study stemmed from a desire to provide a voice for Filipino American women in the health literature, particularly those who share the perceptions and experiences of my mother, who is in midlife. My goal was to gain a greater understanding of the health concerns I could face when I reached her current age. However, I was concerned that, because of my age, my participants may not feel that their experiences would be representative of their age, but rather through my own interpretations. Van Maanen (1988, as cited in O'Bryant, 1996) describes the "realist tale" in the sense that an author plays a large yet invisible role in focusing the limelight on the participants of a study.

In this study, I tried to limit my "personal experiences" during the interview process only as small examples of health beliefs and health care perceptions and to act merely as a collector of the participants' responses, while occasionally clarifying any questions they had. Once I completed the transcription and began coding their data collectively, I attempted to focus solely on their responses and on the emerging themes as they related to the constructs of the health belief model. I also chose to include excerpts directly from the interview transcripts to provide more realistic examples as they relate to the emerging themes or discrepancies (O'Bryant, 1996). In Chapter 4, I outlined those emergent themes and trends relevant to the experiences of 
midlife Filipino American women and their health beliefs in regards to cardiovascular disease and health care utilization.

During the process of data analysis, I was already reflecting on the issues and concerns, as well as garnering unique examples of self-efficacy that each woman spoke of. Even during the interviews, I had made verbal notes to myself regarding the trends I perceived and was able to confirm them with the participants at the time. I transcribed the interviews verbatim while continuing to seek any commonalities or discrepancies among the participants. Additionally, it appeared that I had immersed myself so thoroughly in the background literature that I found commonalities and differences between the sample population and other Asian American groups even before the transcription process was completed.

The biggest challenge I encountered in writing Chapter 4 was how some of the participants' responses to particular questions based on the health belief model did not fit the constructs from which I originally derived them. Some questions that originally fit one construct were better suited to another based on the participants' responses. Nonetheless, I found that these adjustments actually enhanced the trends and themes that had emerged and provided the foundation for the data stories of Chapter 4 (see Appendix D).

\section{Trustworthiness}

Trustworthiness is essential in establishing the legitimacy of a study (Sheurich, 1992, as cited in O'Bryant, 1996). In order to ensure trustworthiness, I implemented triangulation, member checking, and a reflexive research journal. 
Triangulation. Glesne and Peshkin (1987, as cited in O'Bryant, 1996) noted that triangulation is essential in establishing the trustworthiness of an inquiry. Patton (2002) describes triangulation as the use of multiple investigative tools for gathering, analyzing, and presenting data. I included three methods for this study: Face-to-face interviews, member check meetings, and a reflexive research journal.

Member checking. As stated earlier, I utilized an abbreviated form of a member check meeting with each participant via email or telephone inquiries. This was a necessary step in ensuring and confirming accurate representations of the participants in regards to how I portrayed them in the study as well as how I interpreted their responses from the interviews.

Reflexive research journal. Creswell (2003) noted that a "...qualitative researcher...reflects on who he or she is in the inquiry and is sensitive to his or her personal biography and how it shapes the study" (p.182). The conceptual foundations of this research study were based on revelations in the interpersonal relationship between the researcher and an immediate family member. It is because of those interactions that I kept a reflexive journal for conversations, observations, trends, themes, and other unique points that arose in and beyond the scope of the individual interviews.

\section{Ethical Treatment of Participants}

I employed all means possible in protecting the welfare and identities of the participants. This included the administration of an informed consent form (see Appendix C) stating that the participant was aware of any psychological and emotional risks associated with participation in the study. I also applied a pseudonym 
for each participant. Each participant was informed that her participation was voluntary and she had the right to withdraw from the study at any time without penalty.

\section{Summary}

In this chapter, I presented the methods, procedures, and analytical tools used to accurately present the narratives of the health beliefs of four midlife FilipinoAmerican women as they related to cardiovascular diseases and health care utilization. In the next chapter, I provide a description of each participant in the study in regards to her demographic background. In keeping with the purpose of a realist's tale, my perspectives and interpretations are largely absent from the text of Chapter 4 . I organized the data stories that comprise this realist tale around the constructs of the health belief model as well as themes and trends that emerged within in each construct, including an additional exploration into the social factors, as they relate to each participant's health beliefs regarding cardiovascular disease and health care utilization. 


\section{Chapter 4}

Presentation of the Data

The purpose of this study was two-fold. The first was to define the health beliefs of four Filipino American women who were in midlife through in-depth one-on-one interviews regarding cardiovascular diseases and in utilizing health care to prevent, diagnose, or treat those diseases. The second purpose was to conceptualize potential links between the health beliefs of those Filipino American women and their attitudes or choices about health care utilization.

This chapter contains demographic information about the participants as well as their narratives regarding their beliefs of cardiovascular health and health care utilization. I coded the narratives based on the six constructs of the HBM, as described in Chapter 2 . I chose to add another exploratory component, called the social factors, which I believe, would be instrumental in addressing how social influences, such as familial and social networks, may affect health beliefs.

\section{Description of the Participants}

At the time of the interviews, all four participants were between $45-50$ years old. Each participant chose her own pseudonym to which I referred throughout this text as well as during the interview process.

Zsa Zsa

Zsa Zsa, 49, was born in Pampanga and immigrated to the United States, becoming a naturalized citizen in 1982. Her Filipino dialect is Tagalog. She was affiliated with a United States military branch through her husband. Both of her parents were of Filipino descent and she still has relatives in the Philippines. Zsa Zsa 
attended vocational schools in the Philippines before coming the United States, developing a craft in salon services. She utilizes health care services provided by the United States military.

Susan

Susan, 50, was from the Quezon Province and speaks Tagalog. She immigrated to the United States in 1974 and became a naturalized citizen in 1984. Susan's parents were of Filipino descent and she also has relatives in the Philippines. Susan also went to vocational schools in the Philippines before coming to the United States. Although she is a civilian employee for the United States military, she was already affiliated through her husband. She has access and utilizes primary health care provided by the United States military.

\section{Katherine}

Katherine, 50, was born in Manila and speaks Tagalog. She immigrated to the United States in 1971 and became a naturalized citizen in 1984. Katherine was the only participant who did not complete high school or attend college in the Philippines, having moved to the United States and later completing high school and college in California. Katherine's parents were both of Filipino descent. She also has relatives in the Philippines. She has an affiliation with the United States military through her husband and utilizes the health care services provided.

\section{Francisca}

Francisca, 48, was born in the Ilocano region of the Philippines and whose Filipino dialect is Tagalog. Both of her parents were of Filipino descent and she has relatives residing in the Philippines. She immigrated to the United States and became 
a naturalized citizen in 1984. Francisca began college in Manila but moved to the United States and finished at a school in New Mexico. She also had an affiliation with the United States military through her husband but she was the only participant whose primary health care services were not through the military.

\section{Presentation of Interview Responses}

The following section represents participants' responses to questions during the initial interview. I categorized each subsection based on the six constructs of the HBM: Perceived susceptibility, perceived severity, perceived benefits, perceived barriers, cues to action, and self-efficacy. The last subsection was an exploration of social factors.

General Perceptions of Health

I began the interviews with a basic overall inquiry of how each participant defined their perceptions of "good" health and how they recognize changes in their health. My intent was to establish a foundation upon which both the participant and I could build with regards to how they defined their respective health beliefs.

Defining "good health". In general, they all defined the concept of "good health" as an ability to function and be free of illness. Katherine conceptualized it more definitively as being mentally, emotionally, "... and physically able to do everyday stuff without having to feel pain or aches". Susan inferred an aspect of psychological and social balance by including her involvement with the local Filipino club and “...being happy and being with friends". 
Recognizing changes in health. When I asked the participants to describe how their health has changed over the last five years, Katherine was quick to point out that her evolution was both a blessing and a curse. Specifically, she stated that with age, I'm more aware of the need to be healthy, to do work to get healthy...It's gotten worse if you look at it from the outside. But I think I'm healthier now than when I was in my ${ }_{a} 30 \mathrm{~s} . . .[\mathrm{m}] \mathrm{y}$ arthritis is worse now. But in my mind I'm healthier even though I have that disease.

Zsa Zsa noticed changes in her energy levels and that her arthritic condition was worse than in the past. Francisca was diagnosed with a hiatal hernia that had caused a significant amount of pain but stated that, overall, she felt her health was relatively stable. Susan also experienced changes such as being diagnosed with high blood pressure but overall, she felt she had not undergone any major changes that would threaten her health. In general, each participant stated that her current health status was not an inhibiting factor to engage in normal activities.

\section{Perceived Susceptibility}

According to the health belief model (HBM), the construct of perceived susceptibility is, "one's belief regarding the chance of getting a condition" (Janz, et al., 2002, p.49). The following subsections are concepts I extracted that would help me define the participants' perceptions of susceptibility to disease or illness.

Susceptibility to a cardiovascular disease. Susan, Francisca, and Katherine stated that they had a basic awareness of cardiovascular diseases. Katherine was attuned to her susceptibility for high blood pressure because of her parents' medical history and that her older brother also had hypertension. She began taking medications after being 
diagnosed with borderline hypertension but the side effects caused more discomfort than the idea of making adjustments to her diet and physical activity level. She was proud in stating that her decision in making subtle changes in her lifestyle actually lowered her blood pressure.

Francisca's knowledge regarding her susceptibility to cardiovascular disease was based primarily on dietary factors. She mentioned that was a relationship between eating habits and cardiovascular disease. Specifically she associated cholesterol as a risk for heart problems, although she didn't appear to indicate if she knew the differences between "good" and "bad" cholesterol. Susan held similar views about cholesterol in that "...your veins getting clogged with cholesterol...something like that that can cause fat," ultimately connecting unhealthy levels and weight issues with an increased risk in developing a cardiovascular disease.

\section{Perceived Severity}

Janz, et al., (2002) define the construct of perceived severity of the HBM as "one's belief of how serious a condition and its sequelae are" (p.49). I asked the participants to explain how dramatic their lifestyle would change if they found out they had a cardiovascular disease.

Severity regarding a cardiovascular disease. All of the participants shared views on how they would have to change their diet and eating habits, as well as a necessity to lose weight through exercise and increased activity. Mostly they believed in selftreatment options before seeking medical procedures, which all of them did perceive as a very dramatic, if not traumatic, decision. Zsa Zsa was already fairly active but 
would start to watch what she eats. Katherine made an interesting statement in that she would prefer undergoing dramatic changes as motivation:

Right now it's slow change. I'd rather change in a dramatic way because I would be more motivated. I don't like to do but if I have no other choice...that's it...I definitely would change what I would eat, very significantly. And maybe my physical activity would also change dramatically...I would also have to lose weight dramatically; that's one of the things I would have to do very dramatically. Francisca didn't actually quantify in the way that Katherine did but she was willing to make the necessary changes: “...for me, I would be very, very careful. I would change the activities that I have to do in order to live longer. And I would listen to what my doctor has to tell me." Susan was more specific in how it would affect her internally:

... Traumatic change; would have to change your diet and probably have to do a

lot more exercise and so just...that's going to be quite a turn because you're worried about it affecting your heart.

\section{Perceived Benefits}

According to the HBM, the construct of perceived benefits is "one's belief in the efficacy of the advised action to reduce the risk or seriousness of impact" (Janz, et al., 2002, p.49). I explored the participants' perceptions regarding the quality of care they received from their respective health care services. I also examined whether or not there were any advantages in treatment or consultation based on their gender, age, or ethnicity. 
Flexibility of services and perceptions of the health care professional. All of the participants made use of their health care services and providers on a "need-only" basis. Zsa Zsa utilized her services only when there was an unusual issue and Susan went only when necessary. Both Katherine and Francisca understood the importance of yearly check-ups and utilized the services in that manner but also expressed seeking services only when necessary.

In terms of how they perceived their respective health care professionals, all of the participants shared positive experiences including how accommodating and thorough their doctors were in regards to important medical issues and decisions. Zsa Zsa described how her health care professional was proactive in meeting her needs and concerns when she opted for a surgical procedure. Katherine talked about how her health care professional did not force her to make any hasty decisions on the methods of treatment for her hypertension. She felt that his recommendations, which included comparing the positive and negative aspects of self-treatment or oral medication, were invaluable in helping her choose from her options.

Francisca had the privilege of choosing her provider because her doctor was Asian and has since not had any issues with her doctor. Susan shared common perceptions about how thorough her doctor after seeing her general practitioner regarding a kidney issue: she was referred to a kidney specialist after her doctor noticed something in her tests that warranted further investigation. While the specialist confirmed that she did not have any kidney issues, Susan felt that her primary doctor took the necessary steps to assure her that her issues were fully explained. 
Perceptions of gender, ethnicity, and age. I asked the participants about any perceptions they had felt from their health care professionals based on the fact that they were Filipino women nearing or in middle age. I based this question on research noting how some non-Western patients felt their health care provider had been withholding information from them because of differences in any of those three categorizations (Berg, 1999; Ngo-Metzger, et al., 2004; Kennedy, et al., 2005). Surprisingly, none of the participants perceived any gaps or concerns in obtaining information because of those characteristics.

Other positive perceptions of the health care services. I asked the participants to reveal any positive experiences they had during a more recent routine visit to their respective health care providers. Katherine and Zsa Zsa shared common perceptions regarding their health care professionals in that they did not appear to be pressed for time nor did they try to "hurry along" an appointment. Katherine also mentioned the health care system's attempts to improve patients' experiences through phone surveys was another positive aspect because it allowed her to validate her opinions and concerns about her visit.

Francisca enjoyed the fact that she could relate to her doctors and other health care professionals. Her experience with her female doctor was positive, particularly in the fact that the doctor herself returned calls. Susan expressed positive perceptions in regards to availability of the doctors. She emphasized how proactive the personnel were in informing her of when her health care professional changes (as is frequent in the military system). She also mentioned how she felt more confident in the health care professionals because they looked over her records before seeing her as a patient. 


\section{Perceived Barriers}

According to the HBM, the construct of perceived barriers is "one's belief about the tangible and psychological costs of the advised action" (Janz, et al., 2002, p.49). I addressed different perspectives including the participants' perceptions about alternative medicine and any negative experiences with their health care services that may inhibit further utilization.

Non-Western medical perceptions. I addressed non-Western medical perceptions in order to determine if any of the participants were familiar with alternative forms of treatment for any type of ailment. Each participant was clear in indicating their preferences for Western medical practice because of their level of unfamiliarity with non-Western methods.

For example, Zsa Zsa was the only one who appeared very accepting of Western medical practices such as invasive procedures, even noting that she would have it done for "peace of mind". Katherine, Francisca, and Susan also sounded adamant about trying non-Western procedures but were just as hesitant about invasive procedures. For example, all three preferred a "natural" recovery process. Francisca recalled wanting to avoid using steroids for her asthma condition because of the side effects including weight gain and that they made her feel unhappy; Susan was apprehensive about certain medications for similar reasons in that "...they take care of one thing but you will have 500 other things wrong with you".

Katherine made an interesting point in that she believed non-Western medicine would work for people "... who believe it will work". It appeared that she was open- 
minded toward any kind of treatment as long as it was the best and least painful method.

Negative experiences with the health care services. I asked the participants to reveal any negative experiences they had during a routine visit to their respective health care providers. While Francisca was the only participant who couldn't recall any specific details of her last visit, Zsa Zsa, Katherine, and Susan were unique in their experiences and perceptions of their services.

Zsa Zsa discussed an experience when the pharmacy gave her the wrong medications and that she immediately learned to check them before leaving the clinic. Susan mentioned how the revolving door of primary care physicians coming and going was inconvenient and confusing but overall, she couldn't recall any other negative perceptions of the services.

Katherine mentioned slight frustrations in how long appointments had to be set up in advance, especially in that openings were unavailable for a minimum of three weeks. She also relayed one episode wherein she left dissatisfied with her appointment. During that particular appointment, she received a call regarding the status of her husband, who was an ICU patient. Although she told the doctor ahead of time that she couldn't miss the call, she returned to her appointment feeling rushed by the doctor.

\section{Cues to Action and Self-Efficacy}

According to Janz, et al., (2002), the construct of cues to action involve "strategies to activate one's 'readiness" while the construct of self-efficacy was defined as "one's confidence in one's ability to take action" (p.49). I included aspects 
of how the participants gained awareness about the health conditions they were at risk for based on their age and gender. I also asked the participants to describe the types of cues they responded to in finding out more about their current health.

Finding and using resources for current health issues. All of the participants shared a common belief in consulting a doctor for information about their health concerns. Other resources ranged from Zsa Zsa's method of "listening to her body" to reading magazines and doing Internet research. Although I initially chose not to explore their knowledge about menopause, all of the participants appeared to associate diseases other than cardiovascular diseases, such as osteoporosis, with that particular event.

All of the participants stated that they had relative ease in obtaining information about their health concerns. For example, Zsa Zsa has a subscription to a health magazine and Susan uses a book her husband gave her which contains health information. All of the participants also mentioned how the Internet has made it easy for them to obtain information about their conditions.

First exposure to information about cardiovascular diseases. Although all four women had a basic understanding of the differences between cardiovascular diseases and non-cardiovascular diseases, I asked them how they first learned about cardiovascular diseases. Susan heard about them through her doctor while Zsa Zsa read about them in a magazine. Katherine first heard about cardiovascular diseases, specifically hypertension, in a high school biology class as well as at home. Francisca heard about them before but also did not pay attention until recently with blood work she had to get for a non-cardiovascular issue. 
Francisca and Katherine revealed unique perspectives on the connection between cardiovascular diseases and certain milestones, such as pregnancy and aging. Francisca associated discussions relating to cardiovascular diseases as becoming more relevant as one gets older. Yet Katherine mentioned how high blood pressure became a potential issue while she was pregnant in her early 20 's and 30 's. She was told she had high blood pressure during her first pregnancy but when the condition occurred in subsequent pregnancies, she eventually did not perceive the issue as a big concern because she knew it was temporary. She strongly believed that most pregnant women would show signs of hypertension anyway, particularly because of water retention during the final trimester.

Cues to seeking health care services. When I asked the participants about health care utilization, they all shared common perspectives about the events that warranted seeking professional services. I asked them about the types of resources they would use if they found out they were at risk for a cardiovascular disease and all of the participants said they would seek out medical attention immediately if they realized they were at risk for a serious cardiovascular illness. They also shared similar views in the justification of seeking out professional services because of the desire to stay healthy and to avoid deterioration of their health. Zsa Zsa said that she would go "to learn more about the disease" that she could be at risk for, while Susan would go in the hopes of doing something to stop, prevent, or slow down the progression of the disease.

Self-efficacy. It was interesting to note how the participants emphasized the health care services "dual role" in acting as both a confirmatory outlet as well as a resource 
to garner second opinions depending on the severity of the disease. For example, Katherine and Zsa Zsa would continue to self-medicate or heal as naturally as possible for issues that may require surgical intervention. Both preferred to postpone any type of invasive procedure for as long as possible, especially if the disease can be held in check by other means. Francisca readily utilized her health care services for preventative measures in addition to establishing a rapport with her health care professional. Susan was also cautious when it came to surgery but saw the advantage of consulting a health care professional:

...(to) see what advice he would give and then go home and try something natural. I would have two things going on at a time. It will help a lot and slow it down a little bit.

In regards to the participants' interactions with their health care professionals, they all expressed some level of satisfaction in that they were able to express their concerns without feeling inferior. They shared opinions in that their respective professionals were thorough and listened to them as well as making them comfortable and not constrained by time. Katherine and Susan noted that their doctors even volunteered information such as the importance of taking calcium as they enter the menopausal stages. Both Katherine and Susan also believed that if they needed clarification, all they had to do was ask and their health care professional would explain so that they could better understand the issue. Francisca pointed out that her questions were answered before she could ask them: "She's knowledgeable in what she does. Sometimes I don't know what to ask." 


\section{Social Factors}

I added a social aspect to health beliefs based on the literature I found regarding the importance of family and social support in typical Asian cultures and in many non-Western cultures. I also wanted to use this component in the hopes of emphasizing the impact of family and friends in shaping health beliefs, even though it is not a component of the HBM.

The family response. I asked the participants how they thought their family would respond if they had a cardiovascular disease. All of the participants expressed some level of support rather than feelings of neglect or being shunned, but some of them also made a point that support was welcomed to a certain degree. Although Zsa Zsa did not directly address how her family would react if she had a cardiovascular disease, she talked about how she responded to her sister who was recently diagnosed with cancer:

I would be really concerned and I think they (her family) would be the same. They would probably be supportive and we're very close... We're there for each other.

Katherine said her family would be supportive but she emphasized that being supportive was "not positive or negative... I think we tend to be in denial but I think we have accept". When I asked her if she was accepting of their supportive nature she welcomed it but to a point:

You don't tell me what I should do because I have a different way of dealing with things and it's my decision. It's my ailment. Support means you support me in my decision. 
Francisca talked about a medical episode she had and how her husband responded: ...[M]y husband was already dressed up for work. He rushed me to the emergency room and on the way that's when he told his boss that 'I'm not coming to work'...And you know what his supervisor said: 'why can't your wife go to the emergency room?' He [her husband] hang up the phone because he was driving; 'forget you guys; I'd rather lose my job than my wife.'

Susan was certain her family would take the news hard:

That would devastate them. I can't even imagine. The first part they would probably be in denial and then they will be very supportive. Especially my son, he's not good with dying. It would be hard on him.

The role of a social network. To compliment the family role, I asked about how the participants believed a social support group or friends would impact their beliefs about cardiovascular disease. I asked them one particular question in regards to how they would interact with a health care professional if they had a friend or relative present during a consultation. Zsa Zsa, Katherine, and Susan held common views in that they would prefer not to have anyone with them at that time. Zsa Zsa would make exceptions based on the severity of the issue, such as surgery or a lifethreatening situation. Katherine made similar comparisons, noting that only in very serious circumstances, such as having cancer instead of hypertension, would she prefer a friend or relative present:

It wouldn't be comfortable unless it's something grave... But if it's just usual type of diagnosis, there's really no reason to bring a friend or relative. I think it depends on how bad the situation is and I haven't seen anything bad. 
Susan said that she would feel more inhibited and uncomfortable when communicating with her health care provider if another person was present: ...I won't be able to ask the questions I would like to ask... Normally I go by myself. I don't want to talk about stuff with other people around. I just want it to be between me and the doctor.

Francisca welcomed the idea of having a relative present and believed the situation would not interfere with her interactions with the health care professional. In terms of open discussion and having someone else present, she was fortunate to have a health care professional who shared similar characteristics with her:

I don't think [there's a problem] because she's also Asian. With the way we talk, I think she's more open to it. It's nice to have someone in there who has an idea but I don't think she would mind.

I asked an additional question in how the participants viewed consulting their female friends or relatives in order to gain a greater understanding or different perspective of the health concerns they may have or are at risk for. Zsa Zsa embraced the idea of consulting others and was already active in seeking out different perspectives:

Actually I do that with a group of friends and talk about health. You know, I'd rather do that. Even with my clients because maybe I can learn from them and they would learn something from me too... It's really cool actually, you know more.

Francisca was also actively engaged in and valued interaction, especially when she heard a friend had experienced pain from fibroids, a condition from which she was 
also suffering. She found it helpful, although she didn't mention the extent of whether or not her particular friend was Filipino:

I'm relieved now that I have an idea that I might be going through those things too. For now I have something to expect because we have the same problem... [b]ut I would also ask my doctor about it.

Katherine was not as receptive, noting that she didn't “...usually do that. I don't feel any advantage". In terms of communicating with women, she did state that there are issues specific to Filipino women but she wasn't having any at that time and valued any woman's perspective equally, regardless of ethnic background. She also mentioned an important aspect of how dialects can affect communication:

...(I)t's a 'Filipino women' issue but only the people that speak the same dialect. We have many dialects and if it's someone that speaks a different dialect than I speak, I wouldn't understand what they're saying...maybe it doesn't relate because I can't understand them and they can't understand me, unless we do it in English.

Susan's approach was surprising in that she was very subtle in extracting information or consulting others about health concerns:

I would go... when they are talking about it. I would listen in and take it in, and then I would just go and ask 'so what do you do when you have that condition?' not revealing that I also have it. I would ask...hypothetical(ly) but not really tell them that I have something. 


\section{Summary}

In this chapter, I presented the descriptions and narratives of the health beliefs of four midlife Filipino-American women as they related to cardiovascular diseases and health care utilization. Each woman revealed unique health experiences entering midlife, but collectively they shared similar perceptions and beliefs regarding the seriousness of a cardiovascular disease and health care utilization. The health beliefs and perceptions of the Filipino American women who participated in this study were similar to findings in prior research with women from other Asian American groups. However, I did find some differences that warrant further investigation. The next chapter contains an in-depth examination and synthesis of the similarities and differences between participants and includes a comparison to prior research. 


\section{Chapter 5}

Summary, Conclusions, and Recommendations

The purpose of this study was two-fold. The first was to define the health beliefs of four Filipino American women who are in or near midlife, through in-depth one-onone interviews, in regards to cardiovascular diseases and in utilizing health care about those diseases. The second purpose was to conceptualize potential links between the health beliefs of those Filipino American women and their attitudes or choices about health care utilization.

I began this study as a personal quest to provide a voice for Filipino American women in the health literature. In the process, I gained a sense of a group in which various aspects of their health beliefs both matched and differed from other Asian American populations represented in the health literature.

\section{Summary of the Research Process}

In this thesis I sought out midlife Filipino American women to determine their health beliefs in regards to cardiovascular diseases as well as how they utilize health care about those diseases. My overall research question was based on the health belief model: what are the health beliefs of midlife Filipino American women regarding cardiovascular diseases and how do they influence health care utilization?

I developed the following questions based on each specific construct of the HBM as parameters to guide my inquiry in making discoveries addressing the overall question:

1. What do midlife Filipino American women believe about their risks for cardiovascular diseases? 
2. What are their beliefs in the benefits of utilizing health care providers?

3. How do their health beliefs affect health care utilization?

4. How does the familial unit influence attitudes toward health care utilization?

5. How does the social network influence attitudes toward health care utilization?

6. What are the issues that inhibit midlife Filipino American women from confident utilization of medical consultation and available health care resources in the United States?

\section{Discussion}

Before discussing the results of this study, these findings were specific only to the individuals who chose to participate. These findings are not representative of all Filipino American women who are in or near midlife.

Basic Understanding of Health as a Midlife Filipino American Woman Defining "Good Health" in Midlife

The World Health Organization defined health as "a state of complete physical, mental and social well-being and not merely the absence of disease or infirmity" (2006). My intent in asking how each participant defined "good" health was to create a foundation in her perceptions of health of which I then compared through my indirect inquiries (via the interview process) regarding "poor" health. In general, each participant defined good health, in a manner similar to that of the World Health Organization when they described good health, as an ability to function and be free of illness. 


\section{Recognizing Changes in Health}

As noted in Chapter 2, women in midlife experience one major change:

menopause. However, the severity with which that event affects their health varies. The main concerns regarding menopause are the increased risks of osteoporosis and cardiovascular diseases due to decreased hormone levels. I was pleasantly surprised that these women were very attuned to their changing bodies. For example, Katherine stated that her “.... arthritis is worse now. But in my mind I'm healthier even though I have that disease." Similarly, Zsa Zsa noticed changes "energy wise, pain, arthritis, of course", while Susan learned that she had high blood pressure and sensed the need to “...go back to the way I was when I was taking aerobic classes and exercises at home." In a sense, they appeared to not be in denial about change and what the changes might bring, whether they are good or bad.

What do Midlife Filipino American Women Believe about their Risks for Cardiovascular Diseases?

\section{Perceived Susceptibility to a Cardiovascular Disease}

Yee (1997) noted that hypertension is a major concern among Filipinos. As such, this was one of the main reasons I chose to explore the health beliefs about cardiovascular disease. All of the participants emphasized how having the awareness of how cardiovascular diseases manifest played in an invaluable role in preparing them to the possibility of developing one. Katherine was well aware of her susceptibility since high blood pressure was a persistent condition within her family. Francisca appeared to have a basic understanding since she associated dietary factors and cardiovascular disease, mentioning cholesterol in particular. Susan upheld similar 
views with Francisca, regarding negative issues with cholesterol in particular as a risk factor for developing a cardiovascular disease.

\section{Perceived Severity of a Cardiovascular Disease}

All of the participants shared a common perception that a cardiovascular disease would greatly affect their lifestyle patterns but it would also initiate immediate changes. This included making dietary changes and increasing physical activity, two important lifestyle adjustments that they all acknowledged as within their means but nonetheless challenging. They all went into further detail regarding how they would pursue self-treatment such as medication or lifestyle adjustments rather than quick fixes such as surgical alterations. Holroyd, Taylor-Piliae, \& Twinn (2003) alluded to similar beliefs among Filipino immigrant women in Hong Kong in that they would only seek help from a health care system when all other methods of treatment failed. Katherine was interesting in that she would prefer to endure more dramatic issues to motivate changing her ways rather than "slow" issues such as hypertension. Overall, all of the participants expressed that knowledge of being at a high risk for cardiovascular disease would require dramatic, if not traumatic, change.

What are their Beliefs in the Benefits of Utilizing Health Care Providers?

\section{Flexibility of Services and Perceptions of the Health Care Professional}

All of the participants expressed positive experiences with their health care professional overall because they were not forced into any inquiries about their health that might have appeared to be overzealous. The participants were also more receptive to engage in interactions with their health care professional when he or she provided enough information and guidance and did not force decisions given the low 
severity of each participant's respective health issues.

Equally important was how each participant perceived their respective health care professional. For example, Francisca felt fortunate to have a health care professional who was Asian but did not mention any notable differences in how she interacted with her as compared to someone who was not ethnically similar. Her emphasis on having a health care professional who matched her gender, rather than her ethnicity is interesting to note since Cimmarusti (1996) recommended that health care professionals treating Filipino-American patients should consider a "sensitivity" marker wherein a patient's family would either embrace or reject treatment based on how the professional acknowledged cultural values.

Perceptions of Treatment Based on Gender, Ethnicity, and Age

I expected some discrepancies and discomforts based on gender and ethnicity, particularly with participants who were attended by health care professionals who were male and non-Asian. This assumption was based on Kennedy, Taylor and Lee's (2005) findings regarding why some patients change health care providers. The two main reasons they found included poor communication and the inability of the professional to relate culturally and by gender. I'm going to assume that because these women were affiliated with the United States military health care system, they may have become used to seeing a diversity of health care professionals given how military personnel are not usually permanent and don't expect the absence of long-standing relationships to interfere with appropriate health care services. In general, none of the participants perceived any discomforts or biases based on age, ethnicity, or gender. 
Other Positive Perceptions of the Health Care Services

Katherine and Francisca noted how they approved the "proactive" approach regarding the health care systems to which they belonged. Katherine mentioned how she appreciated having the opportunity to discuss her opinions regarding her visits with her health care professional. It appeared important that her viewpoint was acknowledged for improvement or commendation in future visits. Francisca glowed when she mentioned receiving a returned call directly from her doctor. It was as though she was proud that her doctor was one who valued relationships with patients. Susan maintained similar perceptions as Katherine. While there is no known research addressing how proactive health care services shape patients' beliefs in the system, based on the responses of these four women, there may be a possible factor in how their perceptions of service may influence their attitudes toward continued utilization.

How do their Health Beliefs Affect Health Care Utilization?

\section{Cues to Action and Self-Efficacy}

Finding and using resources for current health issues. In contrast to NgoMetzger, et al., (2004) who found that Asian Americans were less likely to report positive experiences with their health care professional, all of the participants expressed how they were receptive to consulting a health care professional about health concerns. However, they did not appear unfamiliar with seeking out other resources if visiting a health care professional would require a waiting period. They were familiar with utilizing the Internet and other forms of media, such as books and 
health-oriented magazines. It would be fair to say that their basic knowledge regarding health issues and beliefs in the health care system do not inhibit them from being proactive in gaining more knowledge about what they might or are at risk for.

First exposure to information about cardiovascular diseases. Each woman gained her first exposure to cardiovascular diseases in unique ways. Katherine was exposed early in life since a cardiovascular condition was present in her family; Susan and Zsa Zsa both gained knowledge through various forms of media and Francisca became more aware after she underwent a blood test for a non-cardiovascular issue. Francisca appeared to be in some form of delayed acknowledgement, stating that she began paying more attention to possible risks as she got older. It appears that their cues regarding cardiovascular diseases were associated mainly by forced means or exposure from family members.

Cues to seeking health care services. When it came to taking an action, all of the participants expressed some level of risk they had to reach before they would consider their status in need of attention by health care professionals. For example, they would seek help health care services immediately if they found out they were at a serious risk for illness but would continue subscribing to treatment methods that are natural or non-invasive until they are no longer effective. As Gelber, McCarthy, Davis, and Seto (2006) noted, there may still be cultural reasons for this "deferred" action.

Self-efficacy. The most common theme emerging from previous sections is that each participant had a belief in her ability to make the necessary changes should her 
risk of cardiovascular disease increase or play a major role in her life choices. They each revealed a surprising association of viewing health care services in a "dual" role: confirming a condition or risk of a condition and as a "second opinion" to which all participants expressed making use of but not discrediting other resources or methods of treatment. Katherine and Susan appeared defiant in agreeing to any invasive procedures as treatment unless all other methods they tried on their own accord failed. Francisca and Zsa Zsa also upheld similar beliefs and confidence in their ability to make necessary changes. This attitude again matches what Holroyd, et al., (2003) found among Filipino women in Hong Kong.

How does the Familial Unit Influence Attitudes toward Health Care Utilization?

\section{The Family Response}

I added this component primarily due to consistent research illustrating how important family connections and cultural cohesiveness are part of the foundations of many non-Western cultures; many cultures continue to stress the importance of including family perceptions for major decisions regarding health. Several researchers have stated that in some Asian cultures, attitudes toward family or community members who have a serious illness range from complete support to complete disassociation. I asked the participants how the presence of a cardiovascular disease would affect the level of support garnered from family members and how the family members would react to them. I found Katherine's perspective unique from the other participants: she appears to have a firm positive belief in her self-efficacy. Although she believed that her family would provide ample support, she would only allow so 
much "pampering", especially if the illness is not immediately life-threatening. In essence, she would not solely rely on health care services based on her family's response. Zsa Zsa expressed how she became very concerned about and supportive of a family member who was diagnosed with cancer and believed the rest of her family, including in-laws, would reciprocate that level of support towards her.

Susan and Francisca appeared to be on the other end of the spectrum, saying that an illness would devastate their families. For Susan, depending on how she perceived the severity of an illness, she believed that even though her family would be very supportive of her, they would also have a hard time accepting what was happening to her. I would make the assumption that Susan would utilize every means possible to find treatments for a serious illness because of the stress she perceived that her family would go through.

Again, based on prior health literature, I did expect more of an accepting and supportive family response. However, I was surprised on how strong-willed these women were when it came to taking care of themselves and understanding that it's fine to have family support but that changes can only start from within. Cimmarusti (1996) noted how mental health counselors should take special consideration of the familial presence but in this case, health care professionals should consider how the person who has the health issue is receptive to treatment. 
How does the Social Network Influence Attitudes Toward Health Care Utilization?

\section{The Role of a Social Network}

I included the social component to gain an understanding of how friends and nonfamily members play a role in how each participant utilizes her respective health care services. One of the questions I included was meant to address how comfort levels between a patient and a health care professional change when a patient's friend or relative was also present during a consultation. Ngo-Metzger, Legedza, and Phillips (2004) found that certain Asian groups have difficulty conceptualizing particular disease because there is no such representation in their culture or language and ultimately were uncomfortable in revealing any issues to a health care professional. I included this inquiry since there was a possibility in linking the presence of another person, particularly a friend or relative who may have a stronger grasp of the language, to act as a mediator, in essence decreasing those perceived discomforts.

I discovered differing viewpoints: Francisca was the only participant who didn't mind having another person present. Zsa Zsa, Susan, and Katherine preferred not having additional company present because they viewed their health care consultation as a private matter and did not perceive discomfort interacting with their health care professional. This may have a lot to do with each woman's ability to establish their take of the issue they are presented without feeling intimidated due to language barriers. 
Additionally, I probed further on how important interactions with social outlets were and how they may impact each participant's beliefs should health related issues arise. It was interesting to find that Zsa Zsa, Susan, and Francisca viewed social outlets as helpful, if not advantageous in finding out the health issues they may have in common. Katherine appeared to be the only participant who felt she could "take it or leave it"; again she asserts her ability to find out things on her own.

Zsa Zsa, Susan, and Francisca had different approaches to those social interactions and based on how I initially perceived their personalities, I wasn't surprised by their responses. Zsa Zsa and Susan struck me as very open and social so it was fitting that their responses were similar: they welcomed opportunities to talk openly about health concerns and learned a great deal talking with friends, even if they did not share common health issues. Susan was more subtle in how she extracted information but she also appeared less inclined to freely discuss her personal health issues, opting instead to present a situation but not admitting that is something she is experiencing. Both Susan and Katherine appeared to be more comfortable absorbing information from afar.

Although I did not explore research that addressed the methods in which people obtain and internalize information, it may be possible that social interactions do play a role in shaping a person's beliefs regarding disease and how he or she chooses to treat the ailment. 
What are the Issues that Inhibit Midlife Filipino American Women from Confident Utilization of Medical Consultation and Available Health Care Resources in the United States?

\section{Perceptions of Non-Western Medicine}

I included an inquiry into the participants' beliefs regarding non-Western medicine because I wanted to get a sense of how knowledgeable and accepting they were with an alternative approach. Since non-Western medicine does not heavily rely on invasive techniques, I was somewhat shocked that all of the participants were not very familiar with it. Because of this unfamiliarity, it made sense that Katherine, Francisca, and Susan were not as receptive to trying it. At this point, Zsa Zsa was blunt in saying that she was more receptive to invasive procedures. However, Katherine did imply that she would not rule it out should all non-invasive Western techniques fail. This discovery matched well with Ford (1997) who noted that a lack of knowledge about a health care resource is one barrier towards its utilization. Additionally, despite Katherine's suggestion of looking to non-Western medicine as a last resort, her perception again coincided with other non-Western cultures' preference in refusing any type of invasive procedures unless there was no other choice (Holroyd, et al., 2003).

Negative Experiences with the Health Care Services

Although each participant did not hesitate to relay a negative experience with her respective health care professional during a prior visit, I did not sense complete dissatisfaction that would have led to avoiding utilization. Zsa Zsa recalled receiving 
incorrect medications but she downplayed its significance, stating that she learned to check what she gets before leaving the pharmacy. Susan was more frustrated with the "revolving door" of physicians but otherwise did not go through any major negative experiences. Katherine detailed a visit in which she felt her doctor was not as understanding when the consultation was interrupted for an important call concerning her husband. However, despite the perception that she was rushed, Katherine did not sound flustered. In the end, she also did not exude any indications of discontinuing to use the services.

\section{Limitations}

\section{Sample Size and Proportion of Participants}

My original goal was to sample at least seven participants. I felt this number would be adequate for gaining perspectives of Filipino American women who were within the proposed age range given my location and access to this population. However, due to the availability and accessibility of prospective participants, I was only able to garner four who met all the requirements as well as having the flexibility of time to meet with me.

\section{Established Criteria for Inclusion}

Several of the established delimitations for this study were very specific and therefore represent only a fraction of a particular population. For example, I chose women who were between the ages of 45 and 55, or were in the average age range of experiencing menopause, as well as making sure both biological parents were native Filipinos. These restrictions limit the diversity and representation of women who may 
have experienced menopause earlier or later or may only have one parent who was a native Filipino.

Variety of Health Care Services and Providers

Because I delimited my sample population to one specific location, I was well aware of the likelihood that all the participants would have access to the same health care system. The women in this study were affiliated with a military branch and were provided health care services through that affiliation.

\section{Conclusion}

So what are the health beliefs of midlife Filipino American women regarding cardiovascular diseases and how do they influence health care utilization? The health beliefs of the four midlife Filipino-American women in this study were firmly established in their self-efficacy in recognizing their risks of cardiovascular disease and in making the best choices to decrease their risk of developing one. In contrast to the assumptions of this study, I found that these women a) were very aware of the resources available to them when they had health issues and b) their health beliefs regarding cardiovascular diseases did not hinder utilization of the health care services to which they had access. In fact, they were more receptive to utilizing health care resources to garner a medical perspective as long as they gained a clearer understanding of the health issue and that the treatment options were as natural as possible.

In regards to their beliefs about the risks of developing a cardiovascular disease, all four women had a basic awareness of their risks, either by personal experience through family members or through utilizing resources that address cardiovascular 
issues. Two of the participants also made links between diet and cardiovascular issues, particularly the effects of "bad" cholesterol on the heart's functioning ability. However, they relied on their ability to make the necessary lifestyle adjustments. Collectively, they were in agreement that cardiovascular diseases are serious but manageable issues. Again, they placed a great amount of self-confidence in their ability to make the necessary changes to minimize their risks without taking drastic treatment measures such as invasive methods.

In terms of the benefits in utilizing health care services, all of the participants emphasized that creating and maintaining positive interactions with their respective health care providers was one major reason to continue use. Based on experiences from prior visits, the participants did not perceive any major issues or causes for avoidance between themselves and their health care provider because of their gender, ethnicity, or age. The participants were also more receptive to continuing utilization when the health care professional made recommendations but left the choice to them. Two participants were very positive in continuing utilization because of how proactive their health care systems were in maintaining or improving effective patient services.

In general, there was no common theme among the participants in terms of how familial perceptions affect health care utilization. However, this is advantageous in illustrating how the decision making process among these women is not reflective of other Asian American societies rooted in patriarchy. For example, one participant does consider her family's viewpoint but ultimately she takes full responsibility for her choices. Two other participants appeared to be heavily influenced by the reactions 
of their families and would include those perceptions when it came to deciding treatment options.

In regards to how the role of a participant's social network influences her views toward health care utilization, each woman placed a different value on its use. For example, two participants who were actively engaged with peers regarding their health status also displayed very outgoing personalities during the interviews. The two remaining participants, who seemed more introverted during their interviews, appeared to subscribe to a less proactive approach that also fit in line with their demeanors. Although this particular aspect can be explored in greater detail, based on the responses from those two participants, the role of the social network did not appear to have a large role in influencing their beliefs in how they utilize their health care services.

Aside from lengthy waiting periods and an avoidance of invasive treatments, there were no major barriers that kept the participants from continuing to use the available health care resources. In regards to their perceptions of non-Western medicine, I was somewhat surprised that none of the women had any in-depth knowledge of that approach but it made sense that they would instead express a preference to continue subscribing to Western medicine because of that level of unfamiliarity.

Unlike some Asian Americans' negative perceptions of utilizing Western-based health care resources, the health beliefs of all four women do not appear to inhibit utilization. Instead, knowing that they have the resources is more a matter of choosing when to utilize them rather than why they should be avoided. For example, all of the women knew how to find and use other resources, especially when seeking out a 
health care professional may require more effort than finding information on their own. However, they did not hesitate to seek out medical attention when they exhausted all the other resources. In regards to receiving treatment, they all remained firmly established in the beliefs that recovery can be done naturally and with as few invasive methods as possible.

Recommendations and Suggestions for Future Research

The following is a list of recommendations and suggestions to gain a broader understanding of the complexities in linking health beliefs and health care utilization within an underrepresented population.

- Compare and contrast military and non-military health care resources.

- Further inquiries regarding health care perceptions should include women who do not have access to health care provided the military or have different health care systems to which they may or may not utilize.

- Compare and contrast women with minor and major cardiovascular health concerns.

- Although the group of women in this study provided invaluable perceptions regarding their health beliefs of cardiovascular disease, their understanding of the seriousness of cardiovascular issues appears optimistic because they have not experienced any significant lifealtering conditions, nor have they had to seek out drastic treatment options. It would be interesting to study the beliefs of women who have experienced such issues. 
- Explore all six constructs as it relates to one specific illness.

- Although the health belief model is an important tool in discovering perceptions regarding behavioral change, it was a challenge exploring health beliefs about a broad range of illness that fall under the categorization of a cardiovascular disease. It may be easier to limit the inquiry to one specific disease and then begin a more detailed investigation of the participant's beliefs about that disease.

- Explore the role of language towards health care utilization in more detail.

- There was evidence in a prior study of how language could adversely affect the decision to seek health care services (Yu, Huang, Schwalberg, Overpeck, \& Kogan, 2002). For example, many members of my extended family have lived in the United States for over 30 years but their accents are still very heavy because they primarily speak Tagalog at home. Given that I struggled to understand their English, it would be imperative to acquire some knowledge on how they might perceive the health care services available to them and their beliefs about illness. I would contend that there are very stark contrasts both in who and how they seek out if they had any health issues as compared to the sample in this study.

- Continue to investigate other cultural factors and health care utilization.

- I did not fully investigate any links between cultural values such as food and health but some researchers have developed connections between Filipino cuisine and an increased risk of cardiovascular 
disease. It would be enlightening to explore health beliefs regarding traditional food preference or other cultural values and the risk of a specific disease, such as heart disease or breast cancer.

- Compare treatment from health care professionals who match the population by gender and ethnicity.

- This would be an excellent control study regarding how a Filipino American or any other non-Western patient would interact with a health care professional who is a match by gender and ethnicity. As prior studies suggest, there may be a perceived level of comfort in which a patient is more willing to disclose personal information to a health care professional because of cultural or biological similarities. 


\section{References}

Allen, J.P. (1977). Recent immigration from the Philippines and Filipino communities to the United States. Geographical Review, 67(2), 195-208.

Araneta, M.R.G. \& Barrett-Connor, E. (2005). Ethnic differences in visceral adipose tissue and Type 2 Diabetes: Filipino, African-American, and White women. Obesity Research, 13(8), 1458-1465.

Arias, E. (2006). United States life tables, 2003. National Vital Statistics Reports, 54(14), 1-40. Retrieved 20 September 2006 from http://www.cdc.gov/nchs/data/nvsr/nvsr 54/nvsr54 14.pdf.

Becker, G. (2003). Cultural expressions of bodily awareness among chronically ill Filipino Americans. Annals of Family Medicine, 1(2), 113-118.

Berg, J.A. (1999). Gaining access to underresearched populations in women's health research. Health care for Women International, 20, 237-243.

Berg, J.A. \& Lipson, J.G. (1999). Information sources, menopause beliefs, and health complaints of midlife Filipinas. Health care for Women International, 20, 81-92.

Brown, C., Matthews, K.A., Bromberger, J.T., \& Chang, Y. (2006). The relation between perceived unfair treatment and blood pressure in a racially/ethnically diverse sample of women. American Journal of Epidemiology, 164(3), 257-262.

Cauley, J.A. \& Danielson, M.E. (2000). Osteoporosis. In M.B. Goldman \& M.C. Hatch (Eds.), Women \& Health (p.1183-1192). San Diego: Academic Press.

Center for Disease Control and Prevention. (2006). Heart disease. Retrieved 20 September 2006 from http://www.cdc.gov/HeartDisease/about.htm. 
Chun, C. \& Sue, S. (1998). Mental health issues concerning Asian Pacific American children. In V.O. Pang \& L.L. Cheng (Eds.), Struggling to be heard (p.75-87). Albany, NY: State University of New York Press.

Cimmarusti, R.A. (1996). Exploring aspects of Filipino-American families. Journal of Marital and Family Therapy, 22(2), 205-217.

Creswell, J.W. (2003). Research design: qualitative, quantitative, and mixed method approaches ( $2^{\text {nd }}$ ed.). Thousand Oaks, CA: Sage Publications.

Devesa, S.S. (2000). Cancers in women. In M.B. Goldman \& M.C. Hatch (Eds.), Women \& Health (p.863-870). San Diego: Academic Press.

Dictionary.com. (n.d.). Retrieved May 13, 2007 from http://dictionary.reference.com/.

Farrales, L.L. \& Chapman, G.E. (1999). Filipino women living in Canada: constructing meanings of body, food, and health. Health care for Women International, 20, 179-194.

Faustino, J. (2003). Perceptions and attitudes of older Filipino Americans toward mental illness. Unpublished doctoral dissertation, Chicago School of Professional Psychology.

Filipinos in the United States. (2003, August). Asian and Pacific Islander American Health Forum. Retrieved 10 May 2007 from www.apiahf.org/resources/pdf/Filipinos_in the United_States.pdf.

Flegal, K.M. (2000). Obesity. In M.B. Goldman \& M.C. Hatch (Eds.), Women \& Health (p.830-838). San Diego: Academic Press.

Flores, P.V. (1998). Filipino American students. In V.O. Pang \& L.L. Cheng (Eds.), Struggling to be heard (p.27-43). Albany, NY: State University of New York 
Press.

Ford, L.D. (1997). Understanding Asian American underutilization of counseling services. Unpublished master's thesis. California Polytechnic State University, San Luis Obispo.

Gelber, R.P, McCarthy, E.P., Davis, J.W., \& Seto, T.B. (2006). Ethnic disparities in breast cancer management among Asian Americans and Pacific Islanders. Annals of Surgical Oncology, 13(7), 97-984.

Gomez, S.L., Kelsey, J.L., Glaser, S.L., Lee, M.M., \& Sidney, S. (2004). Immigration and acculturation in relation to health and health-related risk factors among specific Asian subgroups in a health maintenance organization. American Journal of Public Health, 94(11), 1977-1984.

Harper, M.G. (2006). Childhood obesity. Family \& Community Health, 29(4), 288298.

Ho, P. (2003). Performing the "Oriental": professionals and the Asian Model Minority myth. Journal of Asian American Studies, 6(2), 149-175.

Holroyd, E.A., Taylor-Piliae, R.E., \& Twinn, S.F. (2003). Investigating Hong Kong's Filipino domestic workers' health care behavior, knowledge, beliefs and attitudes toward cervical cancer and cervical screening. Women \& Health, 38(1), 69-82.

Holubkov, R. \& Reis, S.E. (2000). Diagnosis and treatment of heart disease in women. In M.B. Goldman \& M.C. Hatch (Eds.), Women \& Health (p.771-781). San Diego: Academic Press.

How many countries are in the world? (n.d.). Retrieved August 6, 2006 from http://worldatlas.com/nations.htm. 
Janz, N.K., Champion, V.L., \& Strecher, V.J. (2002). The health belief model. In K. Glanz, B.K. Rimer, \& F.M. Lewis (Eds.), Health behavior and health education: Theory, research, and practice $\left(3^{\text {rd }}\right.$ ed) (pp.45-66). San Francisco: Jossey-Boss.

Kaplan-Machlis, B. \& Bors, K.P. (2000). Osteoporosis. In M.A. Smith \& L. Shimp (Eds.), 20 Common Problems in Women's Health care (p.631-663). New York: McGraw-Hill.

Kennedy, H.P., Taylor, D., \& Lee, K.A. (2005). A study of midlife women's reasons for changing health care providers. Journal of the American Academy of Nurse Practitioners, 17(11), 480-486.

Litton, E.F. (1999). Learning in America: the Filipino-American sociocultural perspective. In C.C. Park \& M.M. Chi (Eds.), Asian-American Education: Prospects and Challenges (p.131-153). Westport, CT: Bergin \& Garvey.

Lotufo, P.A., Sabolsi, M., \& Manson, J.E. (2000). Diabetes in women. In M.B. Goldman \& M.C. Hatch (Eds.), Women \& Health (p.819-829). San Diego: Academic Press.

Marshall C. \& Rossman, G.B. (2006). Designing qualitative research ( $4^{\text {th }}$ ed.). Thousand Oaks: Sage Publications.

Nadel, K.L. (2004). Pilipino American identity development model. Journal of Multicultural Counseling and Development, 32, 45-62

National Institutes of Health. (n.d.). Diabetes. Retrieved May 8, 2007 from http://www.nlm.nih.gov/medlineplus/diabetes.html. 
Ness, R. (2000). Cardiovascular disease and cardiovascular risk in women. In M.B. Goldman \& M.C. Hatch (Eds.), Women \& Health (p.753-755). San Diego: Academic Press.

Newton, K.M., Lacroix, A.Z., \& Buist, D.S.M. (2000). Overview of risk factors for cardiovascular disease. In M.B. Goldman \& M.C. Hatch (Eds.), Women \& Health (p.757-770). San Diego: Academic Press.

Ngo-Metzger, Q., Legedza, A.T.R., \& Phillips, R.S. (2004). Asian Americans' reports of their health care experiences: Results of a national survey. Journal of General Internal Medicine, 19(2), 111-119.

O’Bryant, C. P. (1996). Choosing Physical Education as a Profession: Stories of Three African-American Women. Unpublished doctoral dissertation. The Ohio State University.

Oyserman, D \& Sakamoto, I. (1997). Being Asian American: Identity, cultural constructs, and stereotype perception. The Journal of Applied Behavioral Science, 33(4), 435-453.

Patton, M.Q. (2002). Qualitative research \& evaluation methods ( $3^{\text {rd }}$ ed.). Thousand Oaks, CA: Sage Publications.

Pido, A.J.A. (1997). Macro/micro dimensions of Pilipino immigration to the United States. In M.P.P. Root (Ed.), Filipino Americans: Transformation and Identity (p.21-38). Thousand Oaks, CA: Sage Publications.

Porchino, J. (1983). Growing older, getting better. Reading, MA: Addison-Wesley Publishing Company.

Punch, K.F. (1998). Introduction to social research. London: Sage Publications. 
Reichman, J. (1996). I'm too young to get old. New York, NY: Times Books.

Revilla, L.A. (1997). Filipino American identity: transcending the crisis. In M.P.P. Root (Ed.), Filipino Americans: Transformation and Identity (p.95-111).

Thousand Oaks, CA: Sage Publications.

Sharma, M. \& Romas, J.A. (2008). Theoretical foundations of health education and health promotion. Sudbury, MA: Jones and Bartlett Publishers.

Shifflett, B., Buliavac, A., Howd, P., O’Brien, J., \& Seifert, L. (1991). Asians' and Caucasians' purposes for engaging in physical activity. Journal of Sport and Social Issues, 15(2), 145-157.

Shimp, L.A. \& Smith, M.A. (2000). Menopause. In M.A. Smith \& L.A. Shimp (Eds.), 20 Common Problems in Women's Health care (p.91-131). New York: McGrawHill.

Srinivasan, S. \& Guillermo, T. (2000). Toward improved health: Disaggregating Asian American and Native Hawaiian/Pacific Islander data. American Journal of Public Health, 90(11), 1731-1734.

Thomas, J.R., Nelson, J.K., \& Silverman, S.J. (2005). Research methods in physical activity $\left(5^{\text {th }}\right.$ ed.). Champaign, IL: Human Kinetics.

Ursin, G., Spicer, D.V., \& Bernstein, L. (2000). Breast cancer epidemiology, treatment, and prevention. In M.B. Goldman \& M.C. Hatch (Eds.), Women \& Health (p.871-883). San Diego: Academic Press.

US Census Bureau (2002). The Asian population: 2000. Retrieved July 29, 2006 from http://www.census.gov/population/www/cen2000/briefs.html. 
Wong, J.S. (1999). Asian women in sport. The Journal of Physical Education, Recreation, and Dance, 70(4), 42-45.

Wong, M.G. (1980). Model students? Teachers' perceptions and expectations of their Asian and white students. Sociology of Education, 53(4), 236-246.

World Health Organization. (n.d.) About WHO. Retrieved September 12, 2006 from http://www.who.int/about/en/.

Wu, T. \& Bancroft, J. (2006). Filipino American women's perceptions and experiences with breast cancer screening. Oncology Nursing Forum, 33(4), E71E78.

Yee, D. (1997). Issues and trends affecting Asian Americans, women, and aging. In J.M. Coyle (Ed.), Handbook on women and aging (p.14-28). Westport, CT: Greenwood Press.

Yu, S.M., Huang, Z.J., Schwalberg, R.H., Overpeck, M.D., \& Kogan, M.D. (2002). Association of language spoken at home with health and school issues among Asian American adolescents. Journal of School Health, 72, 192-198. 
Appendix A

Background Information Form 


\section{Background Information Form}

Age

What is your citizenship status (Check one)

US Citizen but not born in the Philippines

US Citizen and born in the Philippines (complete the following)

Year Immigrated

Year Naturalized

Not a United States Citizen

Parent's Information (Check one)

B_oth parents were/are Filipino

One parent was/is Filipino

Neither parent was/is Filipino

\section{Health Coverage Profile (Check one of the following)}

Military spouse/member in household (Check one below)

Health coverage through the military

Health coverage but not through the military

No health coverage

No military spouse/member in household (Check one below)

Currently have health coverage

No health coverage

\section{Contact Information}

Name Confuntal

Phone Number Confidenis 
Appendix B

Interview Guide 
The interview will begin with a description of the interview process. In order to protect your anonymity, you have two choices: you may choose a pseudonym yourself or I can choose one for you. I will use this pseudonym during the course of the interviews and in all written transcripts, data analysis, and presentation of results. If you do not feel comfortable answering a question or would me to turn off the recorder, please let me know. Do you have any further questions? Let's get started.

I am interviewing [pseud: on [date at [time .

Demographic Questions

1. What region of the Philippines were you born in?

2. Describe the period of years you attended school in the Philippines. In other words, how long did you attend school there?

a. If you attended college, where did you go?

b. Do you have relatives in the Philippines?

3. When did you last visit the Philippines?

a. How long was your last visit?

General Health Perspectives

1. How do you define" good health"?

2. Describe your beliefs regarding non-Western medical approaches (holistic approach, non-invasive, etc)

Knowledge about the risk of illness Tell me a little bit about your current health.

1. In what ways has your health changed over the last 5 years?

2. How has your health kept you from normal activity?

3. What resources do you use to keep up with current health issues?

4. How much familiarity do you have regarding information about the conditions you might be at risk for as a midlife woman?

5. Describe the difficulties or ease you experienced in obtaining info about the conditions you CURRENTLY have or might be at risk for

Cardiovascular Diseases

1. What do you know about cardiovascular diseases?

2. How did you find out about CV diseases?

3. How dramatic would your lifestyle change if you had a CV illness?

4. If you found out you were at a high risk for a CV illness, would you seek medical attention immediately?

a. Why or why not?

5. If you found out that you developed a CV illness, what resources would you seek out for treatment?

6. How do you think your family would respond if you had a CV illness?

7. If you found that you had a CV illness without consulting a health care professional, would you seek one out if you needed treatment?

a. why or why not? 
Views on Health care Utilization

1. How often do you use the health care services provided for you?

2. Describe your last visit:

a. How did the health care professional accommodate your needs?

b. What concerns did you have that you felt the health care professional could not address with you because of your ethnicity? '

c. Your gender?

d. Your age?

e. How would bringing a friend or relative affect your comfort with the health care professional who tended to you?

f. If you asked questions, how thorough was the health care professional in answering them?

3. How would you feel about consulting people outside your health care network, such as other Filipino women or female relatives regarding the health concerns you have?

4. Describe, in general, your overall experiences with your health care professional/services.

a. What are some positive aspects of the services?

b. What are some negative aspects of the services?

Thank you very much for taking time to talk with me. At this time I would like to remind you that I will contact you for a second meeting (follow-up or member check). Do you have any further questions for me?

NAME 
Appendix C

Informed Consent Form 


\section{INFORMED CONSENT TO PARTICIPATE IN A QUALITATIVE EXPLORATION OF HEALTH BELIEFS AMONG MIDLIFE FILIPINO AMERICAN WOMEN IN CALIFORNIA}

A research study on health beliefs among midlife Filipino American women is being conducted by Lorneneth Merana of the Kinesiology Department at Cal Poly, San Luis Obispo. The purpose of this study was two-fold. The first is to study the health beliefs among a group of midlife Filipino American women (ages 44-55) in regards to cardiovascular diseases and in utilizing health care about those diseases. The second purpose was to ultimately conceptualize potential links between the health beliefs of Filipino American women and their attitudes or choices about health care utilization.

You are being asked to take part in this study by participating in a face-to-face interview with the researcher. Your participation in this interview will take approximately 1-2 hours. The researcher will then request a follow-up meeting, also called a member check meeting. The member check meeting is designated as an opportunity for you to verify or check that the researcher's transcription of the interview is representative of your responses. This meeting may vary in duration from 30-60 minutes.

Please be aware that you are not required to participate in this research and you may discontinue your participation at any time without penalty. You may decline to answer any questions during the interview with which you feel uncomfortable. The researcher will record the interviews with a digital audio recorder. You may request the researcher to turn the recorder off at any point in time that you wish to make comments off the record.

The possible risks associated with participation in this study include psychological discomfort in disclosing personal and possibly medically-related information. If you should experience any emotional or psychological distress as a result of disclosing sensitive information, please be aware that you may contact the researcher's academic advisor, Dr. Camille O'Bryant at 756-1787 for assistance.

Your confidentiality will be protected by the researcher through the use of a pseudonym that you choose for yourself or one that the researcher assigns to you. The researcher will provide pre-interview instructions, including the fact that she will not use your real name any time during the interview. The potential benefits associated with the study include gaining a better understanding of the Filipino American female health perspective as well as the opportunity to share your experiences with a member of the next generation of Filipino American women.

If you have questions regarding this study or would like to be informed of the results when the study is completed, please feel free to contact Lorneneth Merana at 805-704-2134 or Dr. Camille O'Bryant at 805-756-1787. If you have questions or concerns regarding the manner in which the study is conducted, you may contact 
Steve Davis, Chair of the Cal Poly Human Subjects Committee, at 805-756-2754, or Susan Opava, Dean of Research and Graduate Programs, at 805-756-1508.

\section{AGREEMENT TO PARTICIPATE}

I agree to participate in this research study. The researcher provided a thorough explanation of the purpose and background of the study, including the associated benefits and risks that may result due to participation. The researcher also thoroughly explained the interview procedures as well as the expected duration of my participation.

I have been informed that my responses with the researcher will be recorded by a digital audio recorder. My signature below verifies that $I$ have granted permission for the researcher to use these responses. I have been informed that the information I reveal in the interview will remain confidential. I have been informed that the researcher will refer me by my pseudonym during the interview process and in the final manuscript.

I have been completely briefed on the procedures associated and agree to participate in the study. I am aware that I may withdraw from the study at any time without penalty.

I have fully explained to the nature, purpose, and the procedures of the study as described above. I informed the volunteer that she could ask questions before, during, and after the interview, with the digital audio recorder turned off, and explained that I would answer them to the best of my ability. 
Appendix D

Summary Outline of the Trends and Themes 
- General Perceptions of Health

- Good health was defined as an ability to function and be free of illness.

- Current health was not an inhibiting factor to engage in normal activities

- Perceived Susceptibility

- All had a basic awareness of cardiovascular diseases

- Francisca and Susan associated poor dietary habits contributing to risk of cardiovascular disease (CVD)

- Perceived Severity

- Shared views on how they would have to change diet and eating habits, including weight loss through exercise and increased physical activity

- Self-treatment or "natural" recovery processes before invasive procedures

- CVD would cause dramatic changes in lifestyle habits

- Perceived Benefits

- All used their health care services because of flexibility of services in how accommodating their respective health care professionals were

- No perceived discomforts because of age, ethnicity, or gender

- Katherine, Susan, and Francisca expressed being positive in utilizing services because of the system's approach in improving patient services

- Perceived Barriers

- Very little knowledge of non-Western medicine but still great avoidance in invasive procedures

- Treatment preference was overwhelmingly toward natural processes or self-treatment options 
- Negative experiences with the health care services included the revolving door of physicians and having to schedule basic appointments well in advance

- Cues to Action and Self-Efficacy

- Common perceptions in utilizing a health care professional regarding health concerns

- Relative ease in obtaining information about their health concerns from other resources, such as books, Internet, other forms of media

- Cues to seeking out medical attention included an immediate concern for a serious risk of developing a cardiovascular disease

- Justified seeking out health care professionals because of a desire to stay healthy and avoid deterioration of their health

O Health care services also play a "dual role" in acting as a confirmatory outlet and as a resource to garner second opinions of a disease

- All expressed similar levels of satisfaction in that they did not perceive any patient-professional discrepancies when voicing their health concerns

- Social Factors

- Common perceptions regarding familial support should an illness arise, definitely not neglect or disassociation

- Role of the social network was different: Francisca and Zsa Zsa were very active in engaging personal health concerns with other Filipino women, whereas Katherine and Susan were more subtle or cautious in revealing their health issues to others 